PALABRAS CLAVE

Seguridad social

Pensiones

Reforma administrativa

Política social

Igualdad

Chile

Argentina

Rafael Rofman

Especialista Líder en Protección Social,

Oficina Regional para América Latina

y el Caribe, Banco Mundial

œrrofman@worldbank.org

Eduardo Fajnzylber

Profesor de la Escuela de Gobierno,

Universidad Adolfo Ibáñez, Chile

œ eduardo.fajnzylber@uai.cl

Germán Herrera

Profesor de Economía del Sector

Público e Investigador de la Universidad

Nacional de Quilmes, Argentina

œ gherrerabartis@gmail.com
REVISTA CEPAL 101 A AOSTO 2010

\section{Reformando las reformas previsionales: en la Argentina y Chile}

\author{
Rafael Rofman, Eduardo Fajnzylber y Germán Herrera
}

$\mathrm{E}$

n este trabajo se describen las recientes reformas previsionales en la Argentina y Chile. Con ellas se pretendió en los años ochenta y noventa mejorar la sostenibilidad fiscal de largo plazo y el diseño institucional de los sistemas, trasladando parte de los riesgos sociales y económicos desde el Estado a los participantes. En años recientes, las autoridades de ambos países coincidieron en identificar a la insuficiente cobertura entre los adultos mayores y al bajo nivel de los beneficios como los principales problemas de los sistemas previsionales vigentes. Debido a divergencias institucionales y políticas, las respuestas fueron dispares. En Chile, un proceso prolongado y participativo redundó en una amplia reforma concentrada en efectos a mediano plazo mediante ajustes cuidadosamente calibrados. En la Argentina, en cambio, las reformas involucraron un gran número de correcciones sucesivas, con poco debate público sobre sus implicancias y efectos en la cobertura y las necesidades fiscales. 


\section{I}

\section{Introducción}

La Argentina y Chile, dos países pioneros dentro de la oleada de reformas implementadas en los sistemas de pensiones latinoamericanos durante los años ochenta y noventa, se han visto recientemente involucrados en una nueva serie de revisiones y ajustes de sus sistemas jubilatorios. En este trabajo se describen los elementos centrales de las reformas recientes, se explica por qué y cómo estas fueron efectuadas y, al mismo tiempo, se discuten sus potenciales efectos y los desafíos que aún siguen pendientes.

Chile fue el primer país de la región en introducir, a principios de la década de 1980, una reforma estructural de su sistema jubilatorio basado en un mecanismo de capitalización de fondos con gestión privada. El sistema comprendía la participación obligatoria de los asalariados, mientras que los trabajadores independientes podían unirse a él de manera voluntaria. En la Argentina, la reforma de 1993 introdujo un esquema de capitalización similar, aunque no eliminaba por completo el componente de reparto con beneficios definidos. En este sentido, la reforma argentina fue considerada en aquel momento como un avance en relación con la experiencia chilena; tanto el proceso de diseño y discusión política como diversos aspectos técnicos del nuevo sistema fueron considerados más sólidos y sostenibles. ${ }^{1}$

Tal como compartían ciertas características de diseño, los sistemas previsionales de la Argentina y Chile mostraban también algunos problemas básicos comunes. Una baja cobertura, una estructura de costos

\footnotetext{
$\square$ Los autores agradecen la ayuda de David Robalino y los valiosos comentarios y sugerencias de Fabio Bertranou, Carlos Grushka, Hermann von Gersdorff, Mariano Tommasi y un revisor anónimo. Las opiniones vertidas en este documento son de exclusiva responsabilidad de los autores y no representan, oficial o extraoficialmente, las opiniones de los revisores o de las instituciones donde se desempeñan.

Versiones previas de este trabajo fueron publicadas como parte de la serie de Social Protection Discussion Papers del Banco Mundial y presentadas en seminarios y conferencias en Washington, Bogotá y Perugia, Italia. Se agradecen los comentarios recibidos de los participantes en dichos seminarios y conferencias.

${ }^{1}$ Por ejemplo, Arenas de Mesa y Bertranou (1997) indican que el modelo argentino mostraba: “(a)...mayor solidaridad inter e intrageneracional; (b) costos de transición relativamente menores a ser cubiertos por el Estado; (c) una más alta cobertura de los trabajadores independientes; (d) un marco regulatorio más consumado; y (e) menores inequidades de género".
}

administrativos considerada demasiado elevada, un alto nivel de incertidumbre para los participantes y problemas de equidad fueron identificados como las principales limitaciones en ambos sistemas previsionales. El origen de algunos de estos problemas radicaba en la evolución macroeconómica y de los mercados laborales, mientras que en otros casos se vinculaba directamente al diseño de los propios sistemas.

Aunque en los últimos 15 años se produjeron múltiples informes que daban cuenta de los problemas señalados $-\mathrm{y}$ se concretaron algunas acciones puntuales y reformas menores- los cambios de fondo fueron pospuestos, especialmente debido a restricciones macroeconómicas y políticas. La posición fiscal más sólida observada en años recientes, junto con un clima político más propenso a considerar el papel del Estado, creó las condiciones para avanzar en una nueva ola de reformas.

Las reformas en la Argentina y Chile en los últimos años tienen un origen similar. Sin embargo, las medidas de política y los procesos que las sostuvieron fueron diferentes, lo que parece relacionarse con disparidades institucionales y políticas que caracterizan a ambos países. En Chile se sostuvo un debate público con amplia participación, procurándose ampliar el consenso. En la Argentina el camino fue diferente, ya que el proceso político fue limitado, cerrado al debate e instrumentado por medio de decretos o leyes de rápido trámite en el Congreso de la Nación, lo que redujo la discusión sobre objetivos, contenidos y efectos de las reformas propuestas.

Este trabajo se articula en cinco secciones. En las secciones II y III se presenta, para la Argentina y Chile respectivamente, una caracterización del funcionamiento del sistema jubilatorio hacia mediados de la década actual, para luego describir y analizar las reformas recientes, sus potenciales repercusiones y los desafíos pendientes. En la sección IV se discuten las implicancias de los procesos políticos e institucionales, analizando cómo y por qué ciertas características que distinguen a la formulación de políticas (policymaking) de ambos países han influido en aspectos de su política jubilatoria reciente. Por último, en la sección V y final se aportan algunas conclusiones. 


\section{II}

\section{Las reformas en la Argentina}

\section{El escenario jubilatorio argentino hacia el año 2005}

El sistema de pensiones de la Argentina es uno de los más antiguos del mundo y su desarrollo original se remonta a los primeros años del siglo XX. Desde la creación de algunos esquemas ocupacionales, el sistema se expandió hasta que - a fines de los años cuarenta - un fuerte impulso dado por el gobierno peronista redundó en una rápida expansión de la cobertura y, algunos años más tarde, prácticamente todos los trabajadores en la Argentina (incluidos los asalariados y los independientes) se encontraban cubiertos por una serie de esquemas de capitalización parcial relativamente generosos.

Mediante una reforma significativa efectuada a fines de la década de 1960 se integraron los diferentes esquemas y se confirió al gobierno nacional la autoridad para gestionarlos. El nuevo esquema unificado era de reparto, con parámetros comunes. Sin embargo, desde mediados de los años setenta, el sistema enfrentó crecientes problemas financieros y hacia finales del decenio de 1980 resultaba evidente la necesidad de avanzar en una nueva reforma. En 1993, en un contexto de serios cuestionamientos sobre la sostenibilidad fiscal del sistema y debates sobre el papel del Estado en la sociedad, se introdujo una reforma estructural.

En esta sección se discute la situación del sistema hacia mediados de la década actual, considerando aspectos de su diseño y funcionamiento.

\section{Una rápida caracterización del sistema}

Tras la reforma de 1993, el sistema jubilatorio argentino se transformó en un esquema multipilar con componentes de capitalización y de reparto, participación pública y privada en sus mecanismos de gestión, y una combinación de los modelos de beneficios definidos y contribuciones definidas para determinar las prestaciones otorgadas a los jubilados.

Bajo ningún aspecto puede sostenerse que los cambios allí introducidos constituyeron una reforma "definitiva". Desde que la ley original fue sancionada en octubre de 1993, se aprobaron alrededor de 850 nuevas regulaciones referentes al sistema jubilatorio, incluidos 34 leyes y 135 decretos. Aun cuando muchas de estas normas se adoptaron para complementar el diseño original del sistema, existió una clara tendencia a introducir correcciones y rectificaciones de corto plazo.

En el diseño del sistema jubilatorio argentino surgido en 1993 se incluían dos pilares básicos y un esquema de transición. ${ }^{2}$ En el segundo pilar, los trabajadores podían optar entre un esquema de capitalización de fondos con cuentas individuales, gestionado por compañías comerciales privadas, y un esquema de reparto de menor escala, gestionado desde el Estado. Entre las reformas paramétricas más importantes se contaba el aumento de la edad de retiro y del número de años con aportes requerido para acceder a una jubilación. Además, se eliminaban los regímenes especiales que, por distintas razones, establecían condiciones diferenciales para la jubilación de algunas ocupaciones, y se promovía la integración de los esquemas provinciales en el nacional. Finalmente, un sistema complementario de tipo no contributivo proveía un ingreso básico para los adultos mayores pobres.

\section{Evolución reciente}

Luego de la reforma de 1993, la evolución del sistema de pensiones en la Argentina se relacionó estrechamente con las tendencias macroeconómicas imperantes en el país. El creciente deterioro del mercado laboral durante los años noventa hizo sentir su impacto, disminuyendo el número de trabajadores que aportaban al sistema. También cayó el número de beneficiarios (mientras la población adulta mayor aumentaba rápidamente) y el valor de los beneficios se mantuvo casi fijo. Durante la crisis de 2001-2002, los haberes jubilatorios sufrieron un derrumbe en términos reales $\mathrm{y}$, a partir de entonces, propendieron a la recuperación. El escenario fiscal se vio reflejado en la tendencia seguida por los beneficios jubilatorios, debido a que el haber medio constituye el determinante principal del balance financiero del sistema público. Finalmente, la evolución del estado de situación financiera del esquema de capitalización fue marcadamente inestable, a consecuencia de la crisis económica y financiera de principios de siglo y de diferentes ajustes normativos.

${ }^{2}$ Para una discusión detallada sobre sus características, véase Rofman (2003). 
A lo largo de su historia, la Argentina ha sido uno de los países de la región con mayores niveles de cobertura jubilatoria. No obstante, desde los años ochenta, a medida que el desempleo y la informalidad fueron ganando terreno, esta situación comenzó a revertirse. En el gráfico 1 se muestra que el porcentaje de los trabajadores ocupados cubiertos por el sistema descendió desde el 50\%, a principios de los años noventa, a menos del $40 \%$ hacia el año $2003 .{ }^{3}$ Superado el peor momento de la crisis, la cobertura comenzó a crecer y hacia 2006 se acercaba a los niveles observados a fines de la década de 1990. Sin embargo, debe recalcarse que estas tendencias en la evolución de la cobertura no afectaron por igual a los distintos grupos sociales. Mientras que el deterioro general en la cobertura sufrido durante los años noventa, e incluso durante el peor momento de la crisis, no afectó al segmento de mayores ingresos, resultó catastrófico para el quintil de menores ingresos relativos. Entre 1992 y 2003, este último grupo registró una drástica caída de 40 puntos porcentuales en los niveles de cobertura y, a

\footnotetext{
${ }^{3}$ En la información sobre cobertura de activos en la Argentina no se incluye la participación de los trabajadores independientes, por lo que en las tasas de cobertura de activos y ocupados presentadas se subestima el dato exacto.
}

partir de entonces la recuperación fue de tan solo unos cinco puntos.

Mientras que la cobertura de los trabajadores activos disminuyó pronunciadamente durante los años noventa, el impacto en la cobertura de los adultos mayores fue más lento. La relativa baja densidad de contribuciones de muchos trabajadores — según un estudio con datos de 2002, la mayoría de los trabajadores activos en la Argentina tenían historias de contribuciones fragmentadas e incompletas, lo que implica un eventual panorama de exclusión futura de los beneficios jubilatorios- junto con los cambios en la normativa introducidos en los años noventa fueron los principales determinantes (Farall y otros, 2003). La elevación a 30 años del período de aportes requeridos, en un contexto de manifiesto menoscabo del mercado laboral, afectó a muchos trabajadores que se encontraban próximos al retiro. En consecuencia, el número de jubilados bajo el sistema nacional pasó de 2,1 millones hacia finales de 1992 a 1,6 millones en 2005.

En 1992, existían en la Argentina cerca de 80 beneficiarios por cada 100 individuos mayores de 65 años. Esa proporción disminuyó lentamente hasta llegar al $68 \%$ en 2003. Además, la caída no se distribuyó homogéneamente de acuerdo con los niveles de ingreso, siendo mucho más aguda entre los grupos más pobres. En efecto, mientras que los sectores más ricos de la población adulta mayor

GRÁFICO 1

Cobertura de la población activa, ocupada y asalariada, 1992-2009

(En porcentajes)

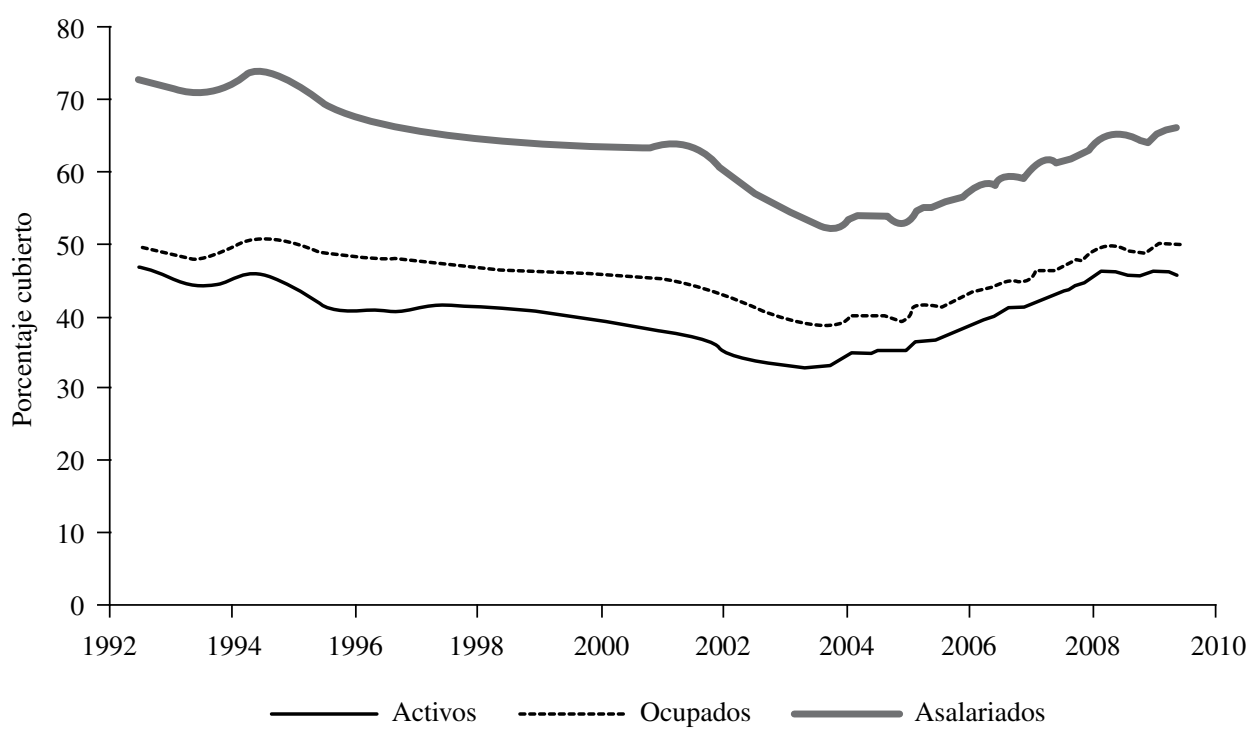

Fuente: Rafael Rofman, Leonardo Lucchetti y Guzmán Ourens, "Pension systems in Latin America: concepts and measurements of coverage", SP Discussion Paper, $N^{\circ}$ 0616, Washington, D.C., Banco Mundial, 2009; Instituto Nacional de Estadística y Censos (INDEC), Encuesta Permanente de Hogares Continua. Primer Semestre 2009, Buenos Aires, 2009. 
no sufrieron un cambio significativo, la cobertura del quintil más pobre se redujo del $63 \%$ en 1992 a un mínimo del $38 \%$ en 2003 (véase el gráfico 2).

En el gráfico 3 se aprecia cómo la evolución de los haberes en los años noventa fue más estable. El haber medio creció lentamente, pero la prestación mínima permaneció sin cambios. Luego del brusco retroceso de los beneficios reales generado por la perturbación (shock) inflacionaria en 2002, el gobierno implementó una agresiva política de incrementos del beneficio mínimo. Promediando el año 2003, este había recuperado ya sus valores previos en términos reales y hacia fines de 2006 superaba en un $65 \%$ el nivel observado cinco años antes. Mientras tanto, los ajustes otorgados al resto

GRÁFICO 2

Cobertura de la población de adultos mayores, total y por quintil de ingresos, 1992-2009

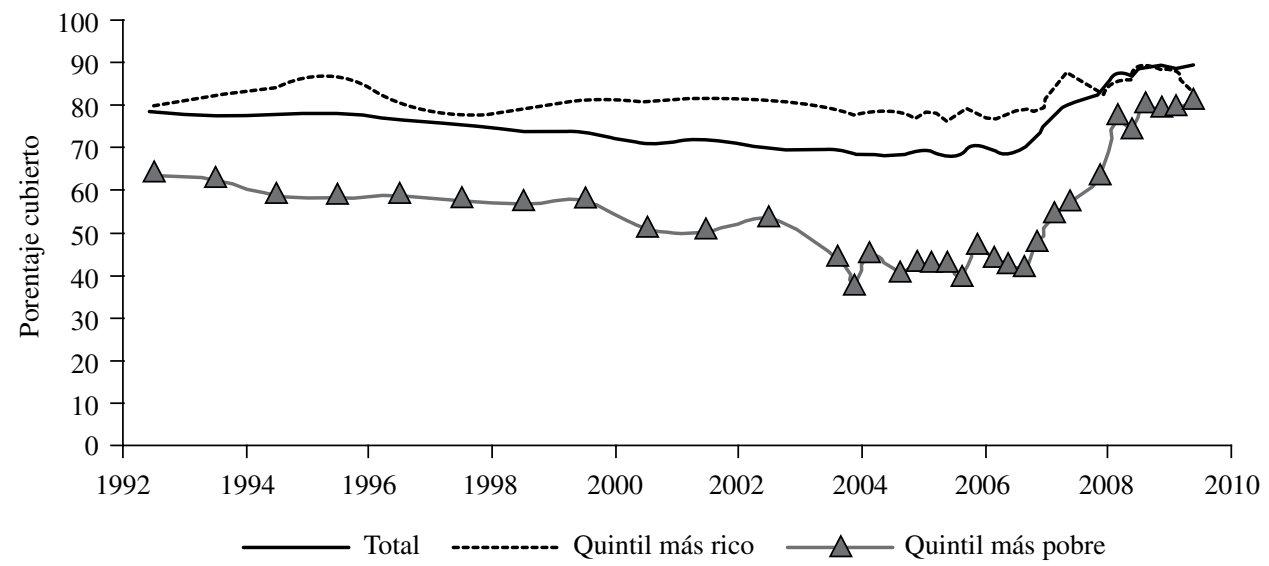

Fuente: Rafael Rofman, Leonardo Lucchetti y Guzmán Ourens, "Pension systems in Latin America: concepts and measurements of coverage", SP Discussion Paper, № 0616, Washington, D.C., Banco Mundial, 2009; Instituto Nacional de Estadística y Censos (INDEC), Encuesta Permanente de Hogares Continua. Primer Semestre 2009, Buenos Aires, 2009.

GRÁFICO 3

Haberes medios y mínimos, y porcentaje de prestaciones en la mínima, 1994-2008

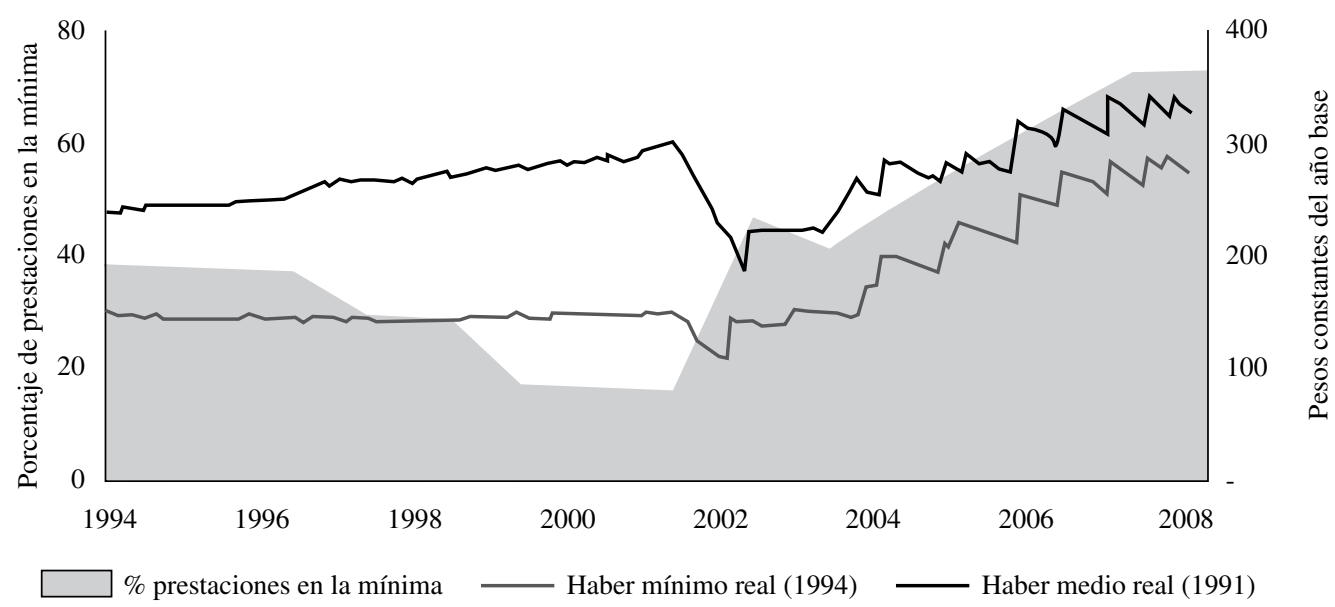

Fuente: Juan Martin Moreno, “Se acuerda, abuelo, cuando las jubilaciones en la Argentina eran Bismarckianas?”, documento presentado en las IX Jornadas Argentinas de Estudios de Población, 2007; Administración Nacional de la Seguridad Social (ANSES), Informe de la seguridad social. Cuarto trimestre 2009, Buenos Aires, 2009. 
de los haberes fueron limitados, con lo que a finales de 2006 el monto del haber jubilatorio mínimo representaba el $83 \%$ del monto del haber jubilatorio promedio. En consecuencia, cerca del $75 \%$ de los beneficiarios del sistema reciben la prestación mínima.

En el gráfico 4 se advierte la tendencia en cuanto a los gastos jubilatorios desde comienzos del decenio de 1980. El muy marcado aumento experimentado entre mediados de los años ochenta y principios de los noventa explica los esfuerzos del gobierno por propiciar una reforma en ese momento. Una vez sancionado el nuevo sistema los gastos totales dejaron de crecer, para luego descender bruscamente con la crisis de 2001-2002 y el congelamiento de los haberes hasta 2005. A partir de entonces se combinaron la recuperación de los haberes con el aumento de cobertura, por lo que en 2008 el gasto estaba en niveles cercanos a los máximos de principios de los años noventa.

\section{Las reformas recientes y sus potenciales repercusiones}

Consideradas en conjunto, las reformas previsionales efectuadas en la Argentina en los últimos años apuntaron a cambiar la cobertura y el nivel de los beneficios del sistema, sus parámetros fiscales, el papel del Estado y del sector privado en cuanto a su gestión, y también algunos aspectos regulatorios del esquema privado. Las reformas se adoptaron mediante distintos instrumentos legales, incluidos varios decretos, leyes y reglamentaciones. Las principales leyes fueron la 26.222, sancionada el 27 de febrero de 2007; la 26.417, sancionada el $1^{\circ}$ de octubre de 2008; y la 26.425, sancionada el 4 de diciembre de 2008. Se podrá notar que, en algunos casos, las normas más recientes afectan a lo determinado por reformas adoptadas con anterioridad. En esta sección se describe con mayor detalle cada uno de estos cambios y se avanza, cuando se considera posible, con respecto a los efectos esperados en el corto y mediano plazo.

\section{a) Cobertura}

Reformas de afiliación para los trabajadores activos

Desde principios de la década, distintas modificaciones derivaron en el progresivo traslado de trabajadores del régimen de capitalización al de reparto. El primer signo de esta tendencia fue la reinstauración de los regímenes previsionales especiales —dirigidos a maestros, investigadores, diplomáticos y empleados judiciales - que fueron eliminados por un decreto en 1994 y que, desde entonces, se habían constituido reiteradamente en materia de litigio judicial. En 2001,

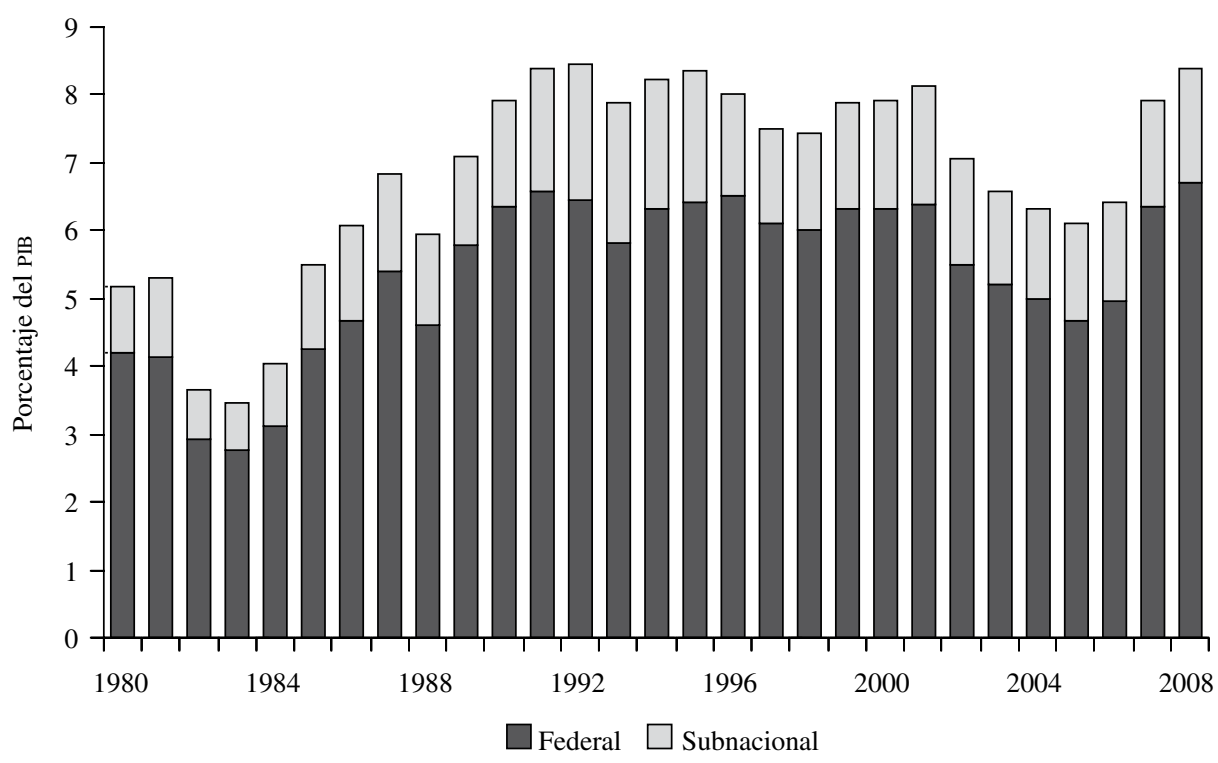

Fuente: Ministerio de Economía y Finanzas Públicas (MECON), “Series de gasto público consolidado”, Buenos Aires, 2009.

PIB: Producto interno bruto. 
las autoridades reimplantaron el esquema especial para diplomáticos y avanzaron sucesivamente con el resto de los regímenes, de modo que en marzo de 2005 los cuatro programas mencionados estaban nuevamente en vigencia. En mayo de 2007 se dispuso que todos los trabajadores pertenecientes a esos regímenes deberían necesariamente dirigir sus aportes al esquema público de reparto y aproximadamente 174.000 contribuyentes - un $1,5 \%$ del total de los afiliados que tenía el sistema de capitalización en ese momento- fueron transferidos a partir de esta disposición (SAFJP, 2007).

Un segundo grupo de trabajadores activos transferidos al sistema de reparto lo integraron aquellos individuos con más de 50 años (hombres) o 55 años (mujeres) que tuvieran menos de $\$ 20.000$ en sus cuentas individuales. La Ley 26.222 establecía que esos trabajadores serían transferidos al sistema de reparto, a menos que manifestaran explícitamente su voluntad de permanecer en el esquema de capitalización. Entre julio de 2007 y marzo de 2008 cerca de 1,1 millones de afiliados fueron transferidos a raíz de esta ley, lo que representaba casi un $10 \%$ del total de afiliados al sistema de capitalización individual (aunque no se dispone de información oficial sobre cuántos de esos afiliados eran, en los hechos, aportantes regulares a sus cuentas). Esta misma ley introdujo, para todo el conjunto de trabajadores, la posibilidad de solicitar el traspaso de un sistema a otro cada cinco años, estableciendo que el primer período para ejercer esa opción permanecería vigente hasta diciembre de 2007. Durante esos meses, casi 1,3 millones de afiliados optaron por pasar del sistema de capitalización individual al sistema público de reparto.

Estas tres medidas en conjunto implicaron que cerca de 2,5 millones de afiliados al sistema de capitalización (el 21\% del total de los afiliados existentes hacia fines de 2006) fueron traspasados al sistema de reparto a comienzos de 2008. Muchos de esos afiliados pudieron haber tenido historias contributivas sumamente discontinuas, pero desafortunadamente no existe información oficial disponible para verificarlo.

Una reforma final fue introducida por medio de la Ley 26.425 en diciembre de 2008, que eliminó completamente el esquema de capitalización, trasladando a todos los contribuyentes a un único sistema público de reparto. En el traspaso se incluyó a los beneficiarios del sistema de gestión privada (a menos que estos estuvieran recibiendo sus haberes a través de una renta vitalicia) y a los activos financieros acumulados.

\section{Reformas de cobertura para los adultos mayores}

Tres grandes acciones fueron implementadas para este grupo de beneficiarios en los últimos años: i) una atenuación de las restricciones de acceso a los beneficios de las pensiones no contributivas; ii) el programa de moratoria previsional, que posibilitó que muchísimos adultos mayores con insuficientes o nulas contribuciones al sistema pudieran retirarse en forma inmediata; $y$ iii) un programa de retiro anticipado.

La Argentina ha tenido beneficios previsionales no contributivos desde hace muchos años. Sin embargo, el acceso a estos beneficios ha sido limitado, tanto en términos de sus condiciones efectivas de obtención (estaban fuertemente racionados y quienes los solicitaban eran asignados a una lista de espera), como del magro nivel de las prestaciones que ofrecían. En tanto, la cobertura del sistema jubilatorio tradicional entre los adultos mayores fue decayendo, y la presión por revisar el esquema no contributivo y hacerlo más accesible fue en aumento.

En marzo de 2003, el Gobierno Nacional creó el Plan Mayores, un programa con el que se procuraba incorporar a mayores de 70 años al esquema del Plan Jefes y Jefas de Hogar. Pocos meses después, en agosto de 2003, la restricción en el número de pensiones no contributivas fue eliminada, lo que condujo a un aumento sostenido del número total de beneficiarios. Así, a fines del año 2006, el número de beneficiarios duplicaba con creces el de tres años antes, mientras que el valor real de los beneficios también se duplicó.

El segundo cambio fue el que tuvo una mayor repercusión, pues introdujo un masivo programa de moratoria previsional. Este programa posibilitaba que todo individuo con la edad de retiro mínima requerida pudiera solicitar un beneficio jubilatorio, "pagando" las contribuciones necesarias para alcanzar los requisitos mínimos del sistema. La ley central de esta reforma fue aprobada en diciembre de 2004, pero hubo -en cuanto a nuevos beneficios otorgados- una respuesta lenta hasta mayo de 2007. A partir de entonces, una rápida aceleración en los procedimientos de aplicación y procesamiento de las solicitudes redundó en un total aproximado de 2,1 millones de nuevos beneficiarios hacia mediados de 2009. En el gráfico 5 se observa cómo el número de beneficiarios previsionales había crecido hasta mediados de los años noventa, para luego estancarse y decrecer. Sin embargo, gracias a la moratoria, el número total de beneficiarios aumentó rápidamente desde 2005.

Finalmente, la tercera reforma que afectó a los niveles de cobertura de los adultos mayores provino 


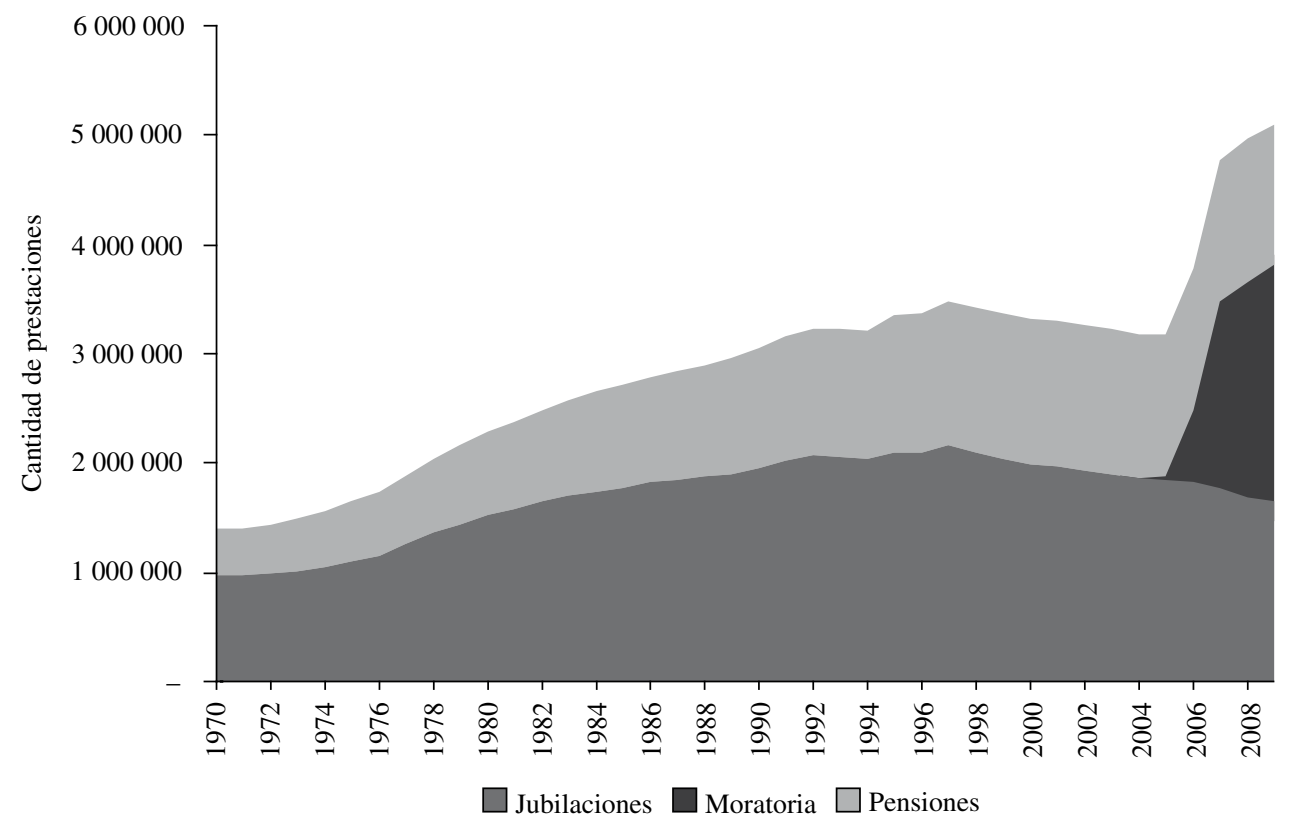

Fuente: Administración Nacional de la Seguridad Social (ANSES), Informe de la seguridad social. Cuarto trimestre 2009, Buenos Aires, 2009.

de la introducción de un esquema de retiro anticipado en diciembre de 2004. Este programa permitió que los trabajadores que hubiesen alcanzado los años mínimos de aportes requeridos, pero que fueran - como máximo- cinco años más jóvenes que lo establecido para hacer efectiva la jubilación, pudiesen hacerlo con una prestación reducida. A diciembre de 2008, aproximadamente 46.000 trabajadores se habían incorporado a este programa.

\section{b) Nivel de los beneficios}

Respecto de las prestaciones previsionales, durante los años recientes se emprendieron acciones en tres áreas. En primer lugar, se incrementaron significativamente los beneficios mínimos; en segundo término, se modificaron los beneficios esperados por los afiliados del sistema de reparto; y en tercer lugar se reintrodujo, luego de 13 años, un mecanismo de indexación automática para los beneficios de reparto.

La principal política con respecto al nivel de los beneficios fue el incremento sostenido del monto de los haberes mínimos y, más recientemente, algún ajuste discrecional en el resto de las escalas de las prestaciones. En el gráfico 3 se mostró cómo los haberes mínimos, en términos reales, crecieron constantemente entre 2004 y 2007. Hacia fines de ese último año, el valor real de la prestación previsional mínima se había duplicado en relación con su valor seis años antes. También fue incrementado el resto de los haberes, pero a un ritmo menor, lo que se tradujo en un achatamiento de la pirámide de prestaciones y debilitó la naturaleza contributiva del sistema.

Por otra parte, entre las reformas introducidas al sistema en 2007 y 2008 se incluyó un aumento de los parámetros correspondientes a la prestación adicional por permanencia. Sobre la base de esta modificación, el componente del beneficio correspondiente a los años con aportes realizados después de 1994 se incrementó en un 76\%.

Finalmente, y en lo que concierne a la indexación de los haberes, después de años de controversias políticas y legales, el gobierno introdujo un mecanismo automático en 2008. En este se establecen dos ajustes anuales en que todos los beneficios del sistema de reparto se incrementan de acuerdo con la evolución de un índice combinado que toma en cuenta el avance de los salarios y de la recaudación de la seguridad social. Asimismo, la ley señala que en el futuro deberá usarse ese mismo índice en el ajuste de los salarios de referencia para calcular el beneficio inicial de los trabajadores que pasan a retiro. 
c) Inversiones de los activos del régimen público

A partir del mejoramiento del resultado financiero del régimen público desde 2003, la Administración Nacional de la Seguridad Social (ANSES) comenzó a obtener un creciente superávit en el manejo de los recursos disponibles. La ANSES recibe fondos provenientes de los aportes y contribuciones de los trabajadores y sus empleadores, así como otros recursos tributarios que le fueron asignados a principios de los años noventa para cubrir el déficit que entonces experimentaba. El superávit se generó por el rápido aumento de la recaudación previsional y tributaria, junto con los ajustes menos amplios para la mayoría de los beneficios. En 2007, el Poder Ejecutivo creó el Fondo de Garantía de la Sustentabilidad cuyo objetivo era administrar estos recursos excedentes. Al transferirse los afiliados del régimen de capitalización al de reparto, los activos correspondientes se depositaron en este mismo fondo gestionado por la ANSES. La Ley 26.425 estableció regulaciones adicionales, incluida la creación de un comité legislativo de supervisión y un consejo con representantes de la sociedad civil. Por su parte, las regulaciones referidas a las políticas de inversión que habrán de ser seguidas a partir de la nueva reforma no fueron explicitadas detalladamente en la ley.

El Fondo mantuvo un bajo perfil hasta fines de 2008, cuando recibió casi 100.000 millones de pesos, cerca de un $10 \%$ del PIB. A partir de entonces, el gobierno ha realizado una serie de anuncios sobre el destino de estos fondos, incluida su inversión en fideicomisos para el financiamiento de consumo, compra de automóviles, financiamiento de pequeñas y medianas empresas, entre otros.

\section{d) Potenciales efectos fiscales de las reformas}

Como consecuencia de los procesos políticos e institucionales bajo los cuales todas estas reformas fueron adoptadas, no ha existido una consideración formal y cuidadosa con respecto a sus potenciales efectos fiscales, ya sea en el corto o mediano plazo. Ninguna de las reformas recientes se adoptó en referencia a una necesidad fiscal y tampoco se sometió el tema a una ronda de presentaciones o de debates públicos. Hasta el momento, ninguna agencia u organismo oficial ha publicado un documento o análisis acerca de las implicancias fiscales de corto y mediano plazo de las reformas, y las declaraciones y referencias realizadas por funcionarios han sido sumamente inespecíficas en esta materia.

Entre las reformas efectuadas, los traspasos del esquema de capitalización al régimen de reparto y la moratoria parecen ser los dos elementos más significativos desde el punto de vista fiscal. Como es obvio, los traspasos implicaron un efecto inmediato en los ingresos del sistema público. En primer término, el traspaso de los saldos de las cuentas individuales a la ANSES, entre 2007 y 2009, involucró el traspaso de activos avaluados en 100.000 millones de pesos, equivalentes a unos dos años de erogaciones del sistema. Sin embargo, la mayor parte de estos activos son ilíquidos, por lo que su disponibilidad en el corto y mediano plazo es limitada. Al mismo tiempo, la incorporación de unos 5 millones de aportantes al régimen de reparto implica una recaudación anual adicional para la ANSES de 18.000 millones de pesos (2\% del PIB).

En cuanto a los gastos, el costo del programa de moratoria se acerca a una cifra equivalente, por lo que en el corto plazo ambas medidas parecerían compensarse. Sin embargo, los efectos en el mediano y largo plazo son menos claros, ya que dependerán de las decisiones de futuros gobiernos sobre la posibilidad de mantener abierto un esquema de moratoria en forma cuasi permanente o cerrarlo ( $\mathrm{y}$, por consiguiente, reiniciar una tendencia declinante en la cobertura).

La construcción de un modelo de proyecciones que permita estimar las tendencias fiscales de mediano y largo plazo relativas al sistema jubilatorio argentino es una tarea dificultosa, pero no imposible y su preparación y difusión debería ser una prioridad de las autoridades. Algunas tendencias parecen claras: el impacto fiscal de la moratoria de 2006-2008 irá en descenso y eventualmente desaparecerá en 15 o 20 años; mientras que los efectos fiscales positivos ocasionados por el cierre del régimen de capitalización deberían compensarse a medida que aumente el número de jubilados en el régimen de reparto. 


\section{III}

\section{Las reformas en Chile}

En enero de 2008, veintiocho años después de la reforma pionera del sistema previsional que reemplazó al tradicional sistema de reparto por uno basado en cuentas individuales, con un mecanismo de capitalización y administración privada, el Congreso Nacional de Chile aprobó la segunda reforma integral del sistema de pensiones. En esta sección se describirá el contexto político y social en que nació esta reforma, se analizarán sus principales contenidos y resultados preliminares y se identificarán algunos de los desafíos pendientes.

\section{El sistema previsional chileno hacia el año 2005}

\section{a) Una breve caracterización del sistema}

El actual sistema de pensiones chileno puede ser desagregado en tres pilares principales: el pilar de prevención de la pobreza, el pilar contributivo y el voluntario.

Antes de la reforma de 2008, el pilar de prevención de la pobreza se basaba en dos componentes: i) el sistema no contributivo de pensiones asistenciales (PASIS) y ii) el sistema de garantía estatal de pensiones mínimas garantizadas (PMG). Este último se orientaba a individuos que, habiendo contribuido al menos durante 20 años al esquema de capitalización individual, no pudieran alcanzar el monto mínimo requerido para jubilarse.

El pilar contributivo fue reformado drásticamente en 1980. El sistema previo se basaba en una serie de esquemas de reparto que otorgaban beneficios definidos, calculados como una proporción de los salarios percibidos durante el último período trabajado. Estos esquemas presentaban crecientes déficits, causados por grandes desbalances entre los beneficios prometidos y las contribuciones realizadas al sistema. En 1980, el gobierno militar creó un esquema nacional único basado en cuentas individuales, donde los ahorros de cada trabajador eran depositados e invertidos en instrumentos financieros por firmas especializadas, las administradoras de fondos de pensiones (el sistema de AFP). ${ }^{4}$ Estas empresas pueden fijar libremente la

\footnotetext{
${ }^{4}$ Solo permanecieron en el sistema previo de reparto los militares y las fuerzas de seguridad.
}

comisión que cobran por los diferentes servicios que proveen (recaudación, registro de las contribuciones, inversiones, cálculo y pago de los beneficios y atención al público) y los individuos pueden optar por cambiar de AFP en cualquier momento. ${ }^{5}$

Con el objetivo de complementar el ahorro obligatorio realizado en el esquema contributivo, por medio de un conjunto especial de productos financieros existen incentivos tributarios a los individuos que realicen contribuciones voluntarias adicionales. Se permite el retiro de los fondos antes de jubilarse, pero quienes lo hagan reciben una penalidad que se suma al impuesto a la renta que debe ser pagado en el momento de dicho retiro.

\section{b) Evolución reciente de la cobertura previsional}

Como el sistema de pensiones chileno se ha basado sobre todo en las contribuciones hechas por los trabajadores asalariados formales, el esquema contributivo es uno de los determinantes más importantes de la cobertura previsional. Desde los primeros esquemas de la década de 1930 , entre el $60 \%$ y el $70 \%$ de la fuerza de trabajo chilena ha estado inscrita en el sistema de pensiones (Arenas de Mesa, 2000). El indicador muestra alguna varianza, dependiendo de los ciclos económicos y las condiciones del mercado laboral. Después de la reforma de 1981, los datos disponibles proveen información sobre los aportantes reales y no solo sobre los trabajadores inscritos. En el gráfico 6 se muestra la proporción de cotizantes en la fuerza de trabajo, que se ha incrementado lentamente en las dos últimas décadas.

Se ha argumentado, sin embargo, que la densidad de contribuciones de los trabajadores, es decir, la fracción de vida trabajada durante la cual una persona realiza aportes sostenidos a la seguridad social, es más importante que la cobertura contributiva. En el gráfico 7 se presenta la distribución de esta medida para los hombres y mujeres chilenos, resaltando el alto grado de heterogeneidad existente en las historias contributivas, que abarcan desde los individuos que contribuyeron durante todo el período

\footnotetext{
${ }^{5}$ Véase una descripción detallada del actual sistema de AFP en Berstein (2007), disponible en www.spensiones.cl. Es amplia la literatura sobre la repercusión en la cobertura de la reforma previsional de 1980, en el desarrollo financiero, en los ahorros nacionales y en el desempeño económico. Véanse, por ejemplo: Corbo y Schmidt-Hebbel (2003); Banco Mundial (1994); Holzmann y Hinz (2005).
} 
GRÁFICO 6

Cobertura contributiva histórica en Chile, 1986-2004

(En porcentajes)

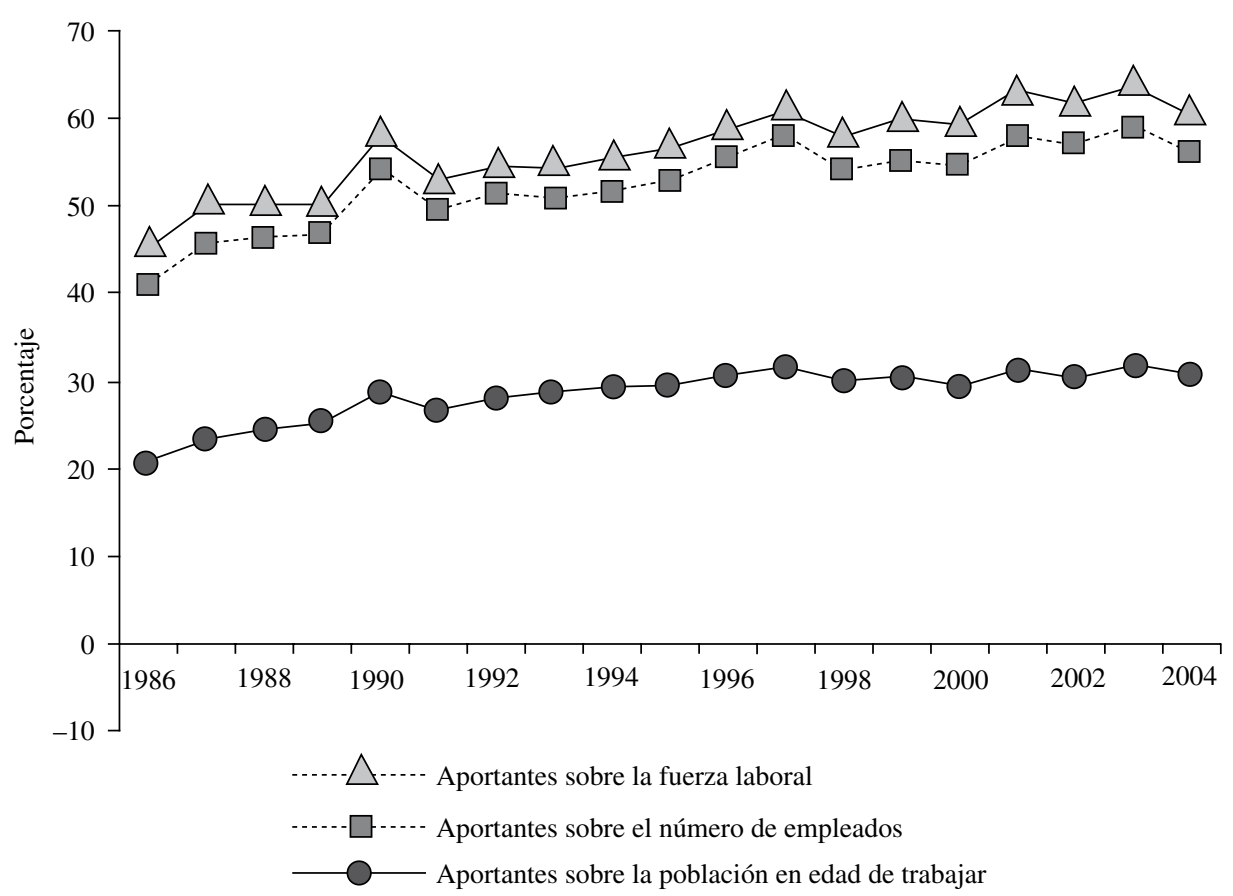

Fuente: Solange Berstein, Guillermo Larraín y Ariel Pino, "Chilean pension reform: coverage facts and policy alternatives", Economía, vol. 6, $\mathrm{N}^{\mathrm{o}}$ 2, Baltimore, Brookings Institution Press, 2006.

GRÁFICO 7

Densidad de las contribuciones al sistema de pensiones (En porcentajes)
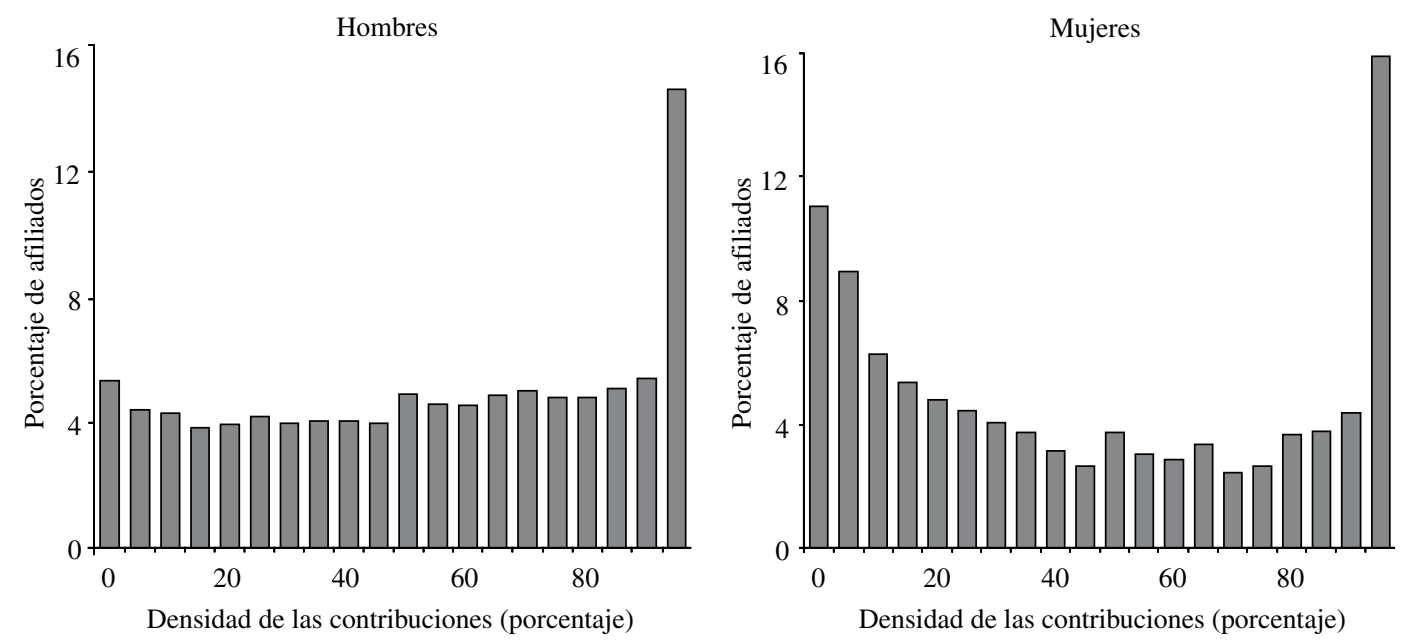

Fuente: Solange Berstein, Guillermo Larraín y Ariel Pino, "Chilean pension reform: coverage facts and policy alternatives", Economía, vol. 6, $\mathrm{N}^{\mathrm{o}}$ 2, Baltimore, Brookings Institution Press, 2006. 
hasta aquellos otros que apenas realizaron contribuciones. Esta heterogeneidad es particularmente notoria entre las mujeres, que muestran una distribución marcadamente bimodal, con una concentración significativa en ambos extremos $(0 \%$ y $100 \%){ }^{6}$

Finalmente, en el gráfico 8 se aprecia la distribución de la cobertura de los adultos mayores en la población chilena. La mitad de la población mayor de 70 años recibe un beneficio del esquema contributivo (actualmente, la mayor parte de esta cobertura es provista

${ }^{6}$ La estimación de las densidades de contribución fue realizada sobre la base de los datos reales para 24.000 trabajadores considerados activos mientras tuvieran edades entre 16 y 59 años. por pensiones de los regímenes de reparto, pero su importancia decrecerá a medida que madure el nuevo sistema). En el gráfico de la derecha se presentan las diferentes fuentes de ingreso para aquellos individuos que no reciben prestaciones directas del esquema contributivo. De esta manera, puede observarse que las pensiones asistenciales, las pensiones por viudez y las pensiones por invalidez proveen algún tipo de cobertura a alrededor del $60 \%$ de este grupo.

\section{c) El clima político: motivos para la reforma}

Una serie de factores pueden haber contribuido a que la candidata presidencial Michelle Bachelet adoptara en 2005 la reforma previsional como una de las

GRÁFICO 8

Fuentes de ingreso en la tercera edad

Individuos con o sin pensión contributiva

(En porcentajes)

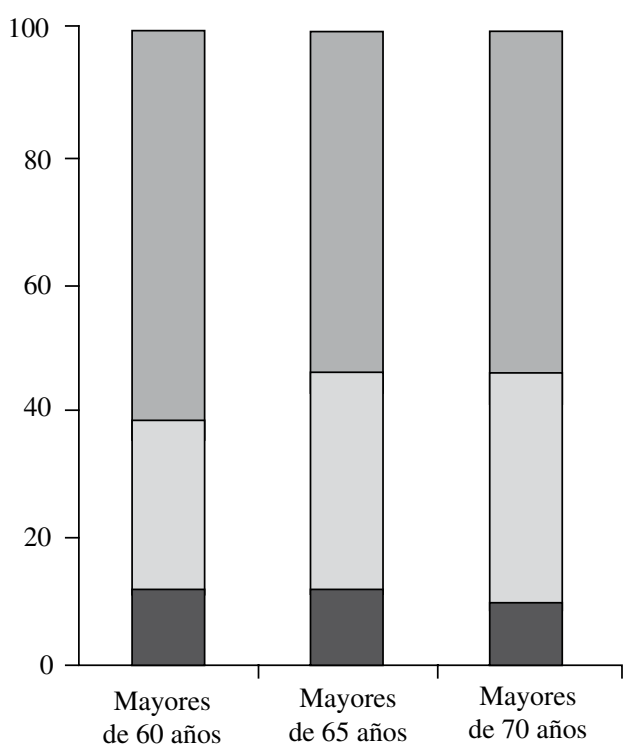

No reciben pensiones contributivas

Reciben pagos del viejo sistema

Reciben pagos de AFP
Individuos sin pensión contributiva (En porcentajes)
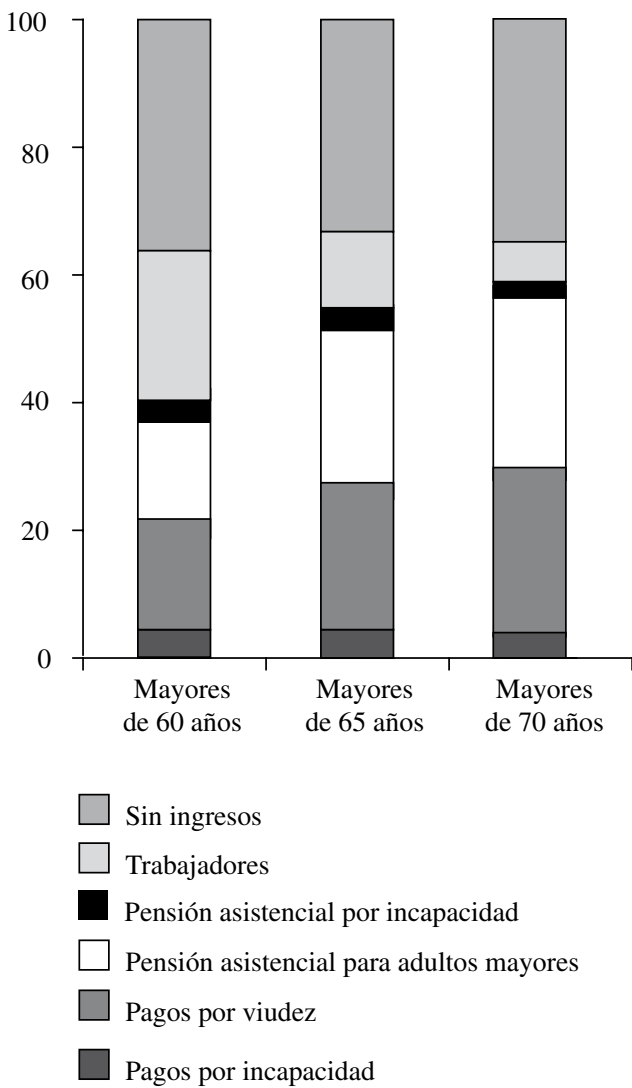

Fuente: Solange Berstein, Guillermo Larraín y Ariel Pino, "Chilean pension reform: coverage facts and policy alternatives", Economía, vol. 6, $\mathrm{N}^{\mathrm{o}}$ 2, Baltimore, Brookings Institution Press, 2006.

AFP: Administradoras de Fondos de Pensiones. 
promesas de campaña más importantes. Desde el retorno a la democracia, la coalición de centro-izquierda había ganado tres elecciones consecutivas impulsando al menos una reforma importante de las políticas o instituciones creadas durante los 17 años de gobierno de Pinochet. La reforma previsional, especialmente de su componente no contributivo, era una de las deudas pendientes de la coalición gobernante. Esta demanda por una reforma que apuntara a mejorar la cobertura se justificaba en parte por los estudios sobre el tema publicados durante los años 2005 y 2006, que sugerían que un gran sector de la población no iba a estar en condiciones de financiar una pensión mínima y no calificaría tampoco para la obtención de la pensión mínima garantizada. ${ }^{7}$

En la decisión de realizar una reforma significativa al componente no contributivo, un segundo factor relevante es el espacio fiscal creado por la reducción gradual en los costos de transición generados por la reforma original de 1980. Como puede verse en el gráfico 9, tanto el déficit operacional vinculado a la eliminación

${ }^{7}$ Véase Berstein, Larraín y Pino (2006) y Arenas de Mesa y otros (2006). gradual del sistema de reparto, como las obligaciones contraídas - mediante bonos de reconocimiento-con los contribuyentes del antiguo sistema que se habían trasladado al esquema de capitalización individual, ya habían comenzado a declinar en 2005. Esto representaba una oportunidad para introducir una amplia red de seguridad social para los adultos mayores.

Por último, existía cierta preocupación en relación con el creciente grado de concentración del mercado de las AFP. En efecto, a pesar de las utilidades sobrenormales sobre activos de las empresas participantes, no se había observado el ingreso de nuevas firmas por un largo período, lo que denotaba que la competencia en precio no estaba funcionando adecuadamente en este mercado en particular. Estos fueron ciertamente algunos de los factores que influyeron en la decisión de la Presidenta Bachelet de avanzar en la reforma del sistema como su principal contribución al desarrollo económico y social de Chile.

\section{La reforma previsional chilena de 2008}

En marzo de 2006, la recién electa Presidenta Bachelet formó un comité presidencial de 15 profesionales,

GRÁFICO 9

Relación entre las pensiones y el gasto fiscal en Chile

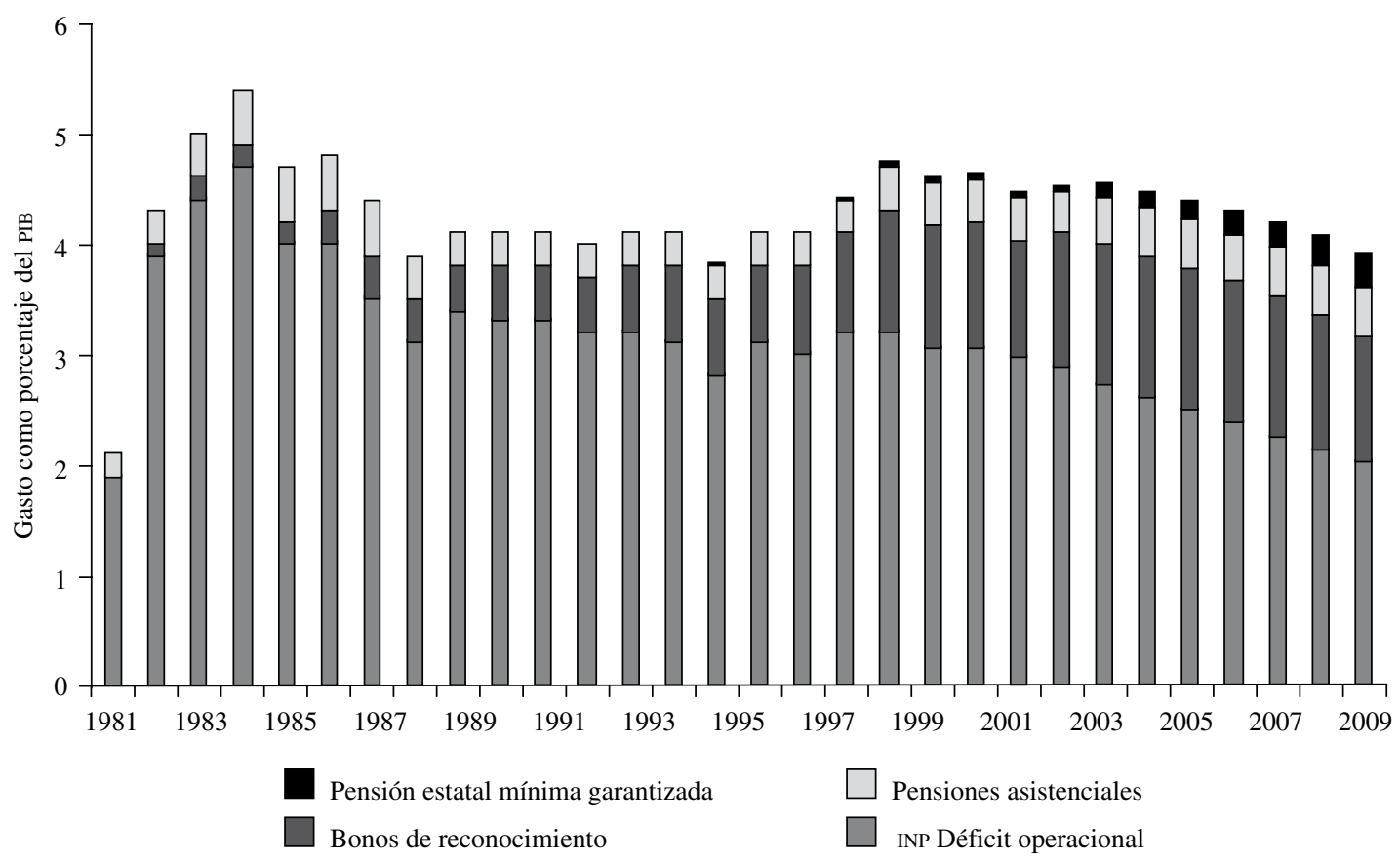

Fuente: Comisión Económica para América Latina y el Caribe (CEPAL), La protección social de cara al futuro: acceso, financiamiento y solidaridad (LC/G.2294(SES.31/3)), Santiago de Chile, febrero de 2006. 
expertos en diferentes áreas relacionadas con el sistema previsional, para redactar un informe con recomendaciones de reforma. ${ }^{8}$ Dos años más tarde, un exhaustivo proyecto de ley fue finalmente aprobado por el Congreso. Esta ley representó la reorganización más significativa desde la reforma original de 1980, que había creado el esquema de AFP. La reforma mantenía la esencia original del sistema, a la vez que introducía mejoras significativas para incrementar la cobertura del pilar de prevención de la pobreza, mejorar la equidad de género, intensificar la competencia en la industria de las AFP e introducir un régimen de inversiones más flexible para las administradoras.

\section{a) Descripción de las reformas}

- Medidas para incrementar la extensión y calidad de la cobertura del sistema previsional

La naturaleza individual del sistema de AFP crea un vínculo directo entre la frecuencia, el plazo (timing) y el monto de las contribuciones realizadas por un individuo y los beneficios que obtiene. Las prestaciones tienden a ser menores cuando los individuos no realizan contribuciones por largos períodos debido a decisiones ocupacionales o informalidad laboral, cuando ingresan tarde al mercado de trabajo formal, o cuando realizan contribuciones que no son proporcionales a su ingreso real. Más aún, los cálculos actuariales sugieren que el incremento de la esperanza de vida requiere mayores ahorros para permitir tasas de reemplazo razonables. Estos pueden generarse mediante un aumento del ahorro voluntario, una vida laboral más extensa o la reducción de los períodos en que se recibe la pensión. La reforma de 2008 aborda estos problemas por medio de una serie de medidas que se describen a continuación.

\section{- El Nuevo Pilar Solidario (NPS)}

La reforma de 2008 reemplaza a los programas existentes: pensiones asistenciales (PASIS) y pensión mínima garantizada (PMG), por un esquema único que garantiza que todos los individuos mayores de 65 años, pertenecientes al $60 \%$ más pobre de la población, tendrán acceso a una pensión básica garantizada independientemente de su historial contributivo. ${ }^{9,10}$ En este nuevo

\footnotetext{
${ }^{8}$ Véase Consejo Asesor Presidencial para la Reforma Previsional (2006).

${ }_{9}$ Para un análisis del pilar de prevención de la pobreza y diseños alternativos, véase Fajnzylber (2006).

${ }^{10}$ El esquema será aplicado gradualmente: en el primer año, a partir de julio de 2008, la Pensión Básica Solidaria equivaldrá a 137 dólares y se restringirá al $40 \%$ más pobre de la población. Este beneficio aumentará a aproximadamente 172 dólares en julio de 2009, cubriendo
}

programa se otorgan subsidios por vejez e invalidez financiados por el Estado.

Los individuos que no hayan realizado aportes tienen derecho a cobrar una Pensión Básica Solidaria (PBS) de vejez si son mayores de 65 años y cumplen con los requisitos de afluencia y residencia. ${ }^{11}$ Los individuos que realizaron aportes, pero que financian una pensión por debajo de un determinado umbral, tienen derecho a recibir un Aporte Previsional Solidario (APS) con los mismos requisitos de afluencia y residencia. ${ }^{12} \mathrm{El}$ programa de invalidez otorga beneficios bajo condiciones similares, pero su población objetivo son los individuos de entre 18 y 64 años. Una vez que los individuos discapacitados cumplen 65 años, son elegibles para los beneficios solidarios de vejez.

En el gráfico 10 se describe el esquema de subsidios, donde los subsidios solidarios y las pensiones totales son presentados como una función de las pensiones contributivas.

Es importante destacar dos elementos de este diseño: la integración entre el sistema contributivo y el Nuevo Pilar Solidario y la preocupación por los incentivos contributivos que esta integración genera. La integración garantiza que en los primeros tres quintiles todos los individuos recibirán una pensión equivalente al menos a la PBS. Si el beneficio se hubiera establecido con un techo (como en las pensiones por invalidez), se habrían creado desincentivos a la contribución por parte de los individuos de bajos ingresos, ya que su pensión no aumentaría con el número o el monto de las contribuciones. Dado el diseño elegido, las pensiones totales por vejez son monotónicamente crecientes con los ahorros financiados por los individuos; cada peso ahorrado incrementa siempre la pensión, aunque la relación no sea de uno a uno.

al $45 \%$ de los individuos de menores ingresos. El esquema de beneficios final entrará en vigencia en julio de 2012 y cubrirá al 60\% más pobre de la población.

${ }^{11}$ La prueba de afluencia es una forma de prueba de ingresos aplicada para determinar que una persona no pertenece al $40 \%$ más rico de la población ( $60 \%$ en el primer año). La implementación inicial (2 años) se realizará sobre la base de la Ficha de Protección Social. La prueba de residencia requiere que los individuos reúnan un período mínimo de 20 años de residencia en Chile, contados desde los 20 años de edad, y de al menos 3 de los 5 años previos a la solicitud del beneficio.

${ }^{12}$ El Aporte Previsional Solidario será pagado primero a aquellos individuos cuyas pensiones contributivas sean inferiores a 161 dólares y pertenezcan al $40 \%$ más pobre de la población en julio de 2008. Este aporte irá aumentando progresivamente hasta el año 2012, cuando el beneficio alcance a aquellos que reciban menos de 586 dólares por medio de sus pensiones contributivas y pertenezcan al segmento del $60 \%$ de menores ingresos. 
Beneficios solidarios de vejez

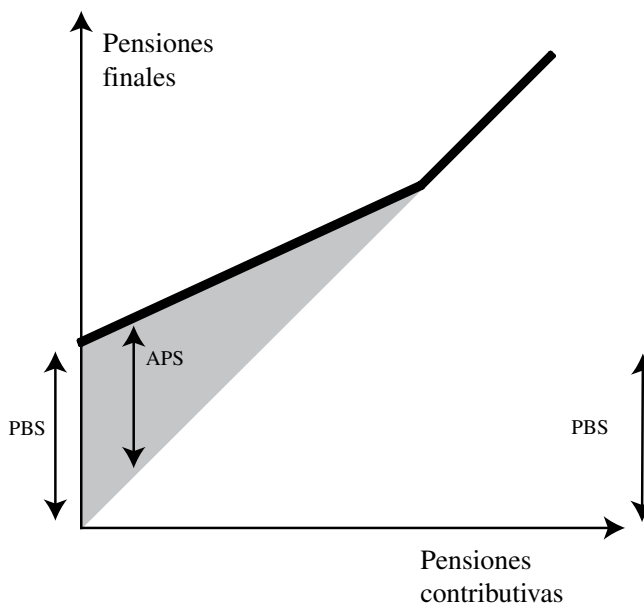

Beneficios solidarios de invalidez

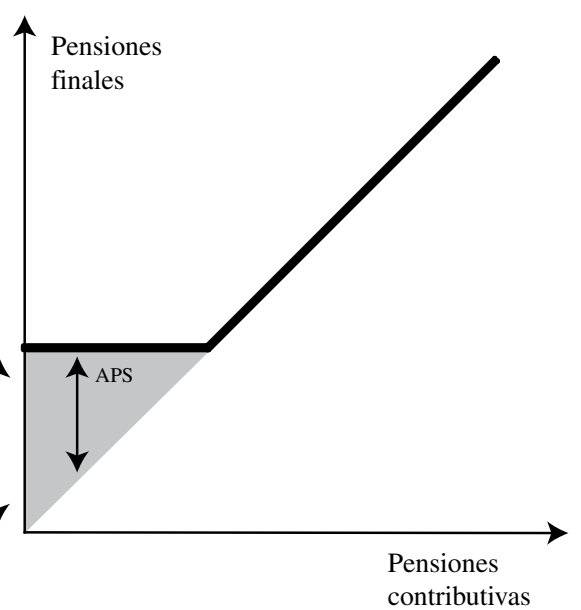

Fuente: elaboración propia.

APS: Aporte Previsional Solidario.

PBS: Pensión Básica Solidaria.

\section{- Contribuciones obligatorias de los trabajadores independientes}

Para ser consistente con la extensión de la cobertura producida por la introducción del NPS, la reforma requiere que todos los trabajadores independientes que reciben ingresos gravados con el impuesto a la renta realicen aportes a la seguridad social sobre sus ingresos anuales. La introducción de este requisito será gradual, comenzando con un período informativo de tres años, seguido por otro período similar durante el cual se descontarán las cotizaciones a los trabajadores independientes, a menos que estos indiquen en forma expresa lo contrario (por defecto, la opción será participar en el sistema). En el curso de este período de transición, la fracción de los ingresos imponible sujeta a este requisito aumentará del $40 \%$ en el primer año, al $70 \%$ en el segundo y finalmente al 100\% durante el tercer año. A partir del año 2015, la participación obligatoria será implementada en su totalidad.

- Planes de Ahorro Previsional Voluntario Colectivo (APVC) e incentivos para los trabajadores de ingresos medios y bajos

En Chile, como en muchos otros países, los ahorros voluntarios para la vejez pueden ser beneficiados con exenciones tributarias. Este tipo de ahorro es conocido como planes de Ahorro Previsional Voluntario (APV).
Los planes de APV pueden realizarse mediante una cuenta especial en una AFP, los fondos mutuos especiales ofrecidos por bancos u otras instituciones financieras, y los contratos de seguro de vida con ahorro. Debido a su diseño, este tipo de exención atrae mayormente a los ahorros voluntarios de los individuos de altos ingresos, que están sujetos a las mayores tasas marginales de impuesto a la renta. Para la mayoría de los trabajadores de ingresos bajos y medios, que no están sujetos al impuesto a la renta, las exenciones impositivas regulares no presentan incentivos para participar de estos planes.

La reforma comprende dos mecanismos para incrementar los ahorros voluntarios de los trabajadores en relación de dependencia, en general, y especialmente los ahorros de aquellos trabajadores que no se hayan beneficiado por las exenciones impositivas regulares. Por una parte, crea la figura de los planes de Ahorro Previsional Voluntario Colectivo (APVC), un esquema que provee incentivos fiscales a las firmas que ofrezcan a sus trabajadores planes de ahorro con aportes complementarios de la empresa. ${ }^{13}$ Por otra, la reforma

\footnotetext{
${ }^{13}$ Los planes de APVC siguen el mismo principio de los planes privados de pensiones denominados $401 \mathrm{~K}$ de los Estados Unidos u otros planes ocupacionales de contribución definida aplicados en otros países. Los empleadores pueden establecer contratos de ahorro con cualquier
} 
considera dos incentivos adicionales para los ahorros voluntarios individuales:

i) Los trabajadores pueden elegir si la exención impositiva se aplica en el momento de realizar los aportes o cuando estos son retirados.

ii) los trabajadores pueden beneficiarse con una bonificación, financiada por el Estado, del 15\% sobre los aportes voluntarios (individuales o colectivos) que se destinen a acrecentar el monto de la pensión, sujeta a un máximo anual, o a anticipar el momento de retiro.

- Subsidios a las cotizaciones previsionales de los trabajadores jóvenes.

Un aspecto particular de los sistemas de contribución definida es que, debido al efecto del interés compuesto sobre un período largo, las contribuciones tempranas pueden tener un efecto significativo sobre la prestación final. Por esta razón, y en aras de reducir el desempleo entre los jóvenes, se crea un subsidio especial para pagar parte de las obligaciones previsionales de los empleadores que contraten trabajadores con edades entre 18 y 35 años. Específicamente, se subsidiará a los empleadores por un monto equivalente al $50 \%$ del costo previsional (aporte más comisión) de un trabajador de salario mínimo, durante los primeros 24 aportes de los trabajadores jóvenes cuya remuneración sea igual o inferior a 1,5 veces el salario mínimo mensual.

Además, dichos trabajadores recibirán una bonificación financiada por el Estado equivalente al subsidio de contratación, que será depositada directamente en su cuenta individual. Esta bonificación regirá para las primeras 24 contribuciones entre los 18 y 35 años, realizadas por un salario inferior a 1,5 veces el salario mínimo.

\section{b) Medidas para mejorar la equidad de género en el sistema previsional}

En la reforma se puso especial atención en introducir medidas que pudieran incrementar la equidad de género. En general, las mujeres tienden a:

i) Pasar largos períodos sin aportes, lo que se relaciona con el cuidado de hijos u otros parientes dependientes.

institución que ofrezca planes individuales de APV (AFP, bancos, fondos mutuos y compañías de seguros); complementar las contribuciones realizadas por los trabajadores; y establecer años mínimos de cotizaciones para acceder a los aportes realizados por la empresa. Las condiciones deben ser las mismas para todos los trabajadores y los empleadores no pueden, bajo ninguna circunstancia, restringir los beneficios a determinados grupos. ii) Ser empleadas en ocupaciones de menor remuneración (en comparación con hombres con similar nivel educativo).

iii) Pensionarse antes que los hombres.

iv) Tener una esperanza de vida mayor. ${ }^{14}$

Todos estos elementos, combinados en un sistema de pensiones que no involucre una redistribución entre sexos durante la fase de retiro, crean diferencias significativas por género.

\section{- Introducción del Nuevo Pilar Solidario (NPS)}

Para abordar estas diferencias, en la reforma se implementa una serie de medidas, la principal de las cuales es la introducción del NPS. En efecto, por su diseño los beneficios solidarios llegarán más frecuentemente a las mujeres, más propensas a no haber realizado nunca aportes o a haberlo hecho con menor frecuencia que los hombres. Además, los beneficios son independientes del sexo de la persona, lo que tiende beneficiar más a las mujeres, usualmente más longevas que los hombres.

- Bono para las madres, financiado por el Estado, por cada hijo nacido vivo o adoptado.

La reforma introduce una bonificación por cada hijo nacido vivo o adoptado. El subsidio equivale al aporte por un salario mínimo de tiempo completo durante 18 meses y es aumentado por una tasa de retorno anual - equivalente al retorno neto promedio de los fondos tipo C del sistema de AFP- desde el día del nacimiento hasta que la madre cumple 65 años. Este beneficio está sujeto al requisito de residencia, pero no a test de ingresos.

Como Chile se encuentra entre los países de la región que presentan los mayores períodos de licencia por maternidad (18 semanas) y al mismo tiempo una de las tasas más bajas de participación femenina en la fuerza de trabajo, la introducción de esta bonificación es importante para alcanzar jubilaciones adecuadas, particularmente entre las trabajadoras de bajos ingresos. Más allá del beneficio financiero, la medida es valorada como una forma de reconocimiento social a las actividades (no remuneradas) de dar a luz y cuidar de los niños durante los primeros meses de vida.

\section{- Compensación económica en caso de divorcio o} anulación del matrimonio.

La reforma introduce el concepto legal de compensación económica previsional en caso de divorcio

\footnotetext{
${ }^{14}$ La edad mínima de retiro es 60 para las mujeres y 65 para los varones. En el informe del Consejo Asesor Presidencial para la Reforma Previsional se recomendó aumentar la edad de retiro de las mujeres a 65 años, pero esta recomendación no fue incluida en el proyecto de ley enviado al Congreso.
} 
o anulación del matrimonio. Bajo esta figura, un juez puede instruir, si es necesario, la transferencia de fondos de pensión entre cuentas individuales, como una forma de compensación económica a la parte que presenta pérdidas durante el matrimonio. Esta transferencia no puede superar el 50\% de los fondos acumulados durante el matrimonio en la cuenta del cónyuge que debe compensar.

- Separación de los contratos de seguro de invalidez entre hombres y mujeres y transferencia de la diferencia de prima a las cuentas individuales del grupo de menor costo.

Previo a la reforma, la prima cobrada por el Seguro de Invalidez y Sobrevivencia (sIs) a los participantes del sistema de AFP era la misma para hombres y mujeres, a pesar de que es menos probable que estas se conviertan en discapacitadas y no generen beneficios de sobrevivencia para sus esposos, a menos que estos sean discapacitados. Para evitar este subsidio cruzado, en la reforma se requiere que las AFP suscriban contratos de seguro separados para hombres y mujeres, que cobren a los afiliados la mayor de las nuevas primas (más probablemente aquella ligada al contrato de los hombres) y depositen la diferencia respecto de la prima inferior en las cuentas de ahorro del grupo de menor riesgo (con mayor probabilidad, las mujeres). Como resultado, la contribución final de las mujeres a sus fondos de pensión será ligeramente mayor que el 10\% indicado en la ley. Esto puede ser visto como una manera de mantener un costo único del seguro para todos los participantes, a la vez que se incrementa el monto de ahorro disponible para las mujeres en el momento de la jubilación.

\section{- Pensiones para los viudos.}

Una de las principales asimetrías de género prevaleciente en el sistema previsional es la imposibilidad de generar pensiones de sobrevivencia para los viudos, a menos que sean discapacitados. Como parte de la reforma, tanto el requisito de reservar una parte de los fondos de pensión acumulados para pagar pensiones de sobrevivencia, como la cobertura bajo el seguro de sobrevivencia son aplicables tanto a hombres como a mujeres. En el primer caso, la inclusión de los viudos disminuirá actuarialmente la pensión de las mujeres jubiladas a cambio del beneficio adicional. En el segundo caso, la cobertura adicional será financiada por una prima de seguro única correspondiente a todas las mujeres del sistema, eliminando así los actuales subsidios cruzados desde las mujeres aseguradas hacia los hombres asegurados.

Las medidas aquí descritas dan cuenta del mayor número de las acciones que pueden implementarse para mejorar la equidad previsional entre hombres y mujeres a través del diseño del sistema de pensiones. Sin embargo, gran parte de la desigualdad previsional se relaciona con factores culturales que rigen la distribución del trabajo en el interior de los hogares y con distorsiones del mercado de trabajo que operan a través de la discriminación ocupacional o salarial. Estos factores no pueden ser abordados apropiadamente por medio de reformas a los sistemas de pensiones.

c) Medidas para incrementar la competencia en el mercado de las $\mathrm{AFP}^{15}$

Uno de los principales pilares de la reforma de 1980 fue la introducción de la competencia entre las AFP como mecanismo disciplinador de las administradoras, a objeto de asegurar buen desempeño, buena calidad del servicio y a un bajo costo. La realidad ha demostrado que la competencia en una industria donde el servicio es obligatorio y extremadamente complejo para el consumidor medio y en que los beneficios solo se perciben en el largo plazo, no siempre adopta la forma deseada. En los últimos años, se ha observado una tendencia a la concentración del mercado (cinco firmas administran fondos equivalentes al $60 \%$ del PIB), altos retornos de las administradoras sobre sus activos y ausencia de entrada de nuevos competidores en los últimos nueve años. ${ }^{16}$

- Licitación de afiliados.

La reforma aborda estos problemas influyendo tanto en la demanda como en la oferta del mercado. ${ }^{17}$ Por parte de la demanda, la elasticidad se incrementa sustancialmente mediante la introducción de un proceso de licitación de afiliados. Todos los nuevos participantes del sistema de pensiones serán automáticamente afiliados en la AFP que haya ofrecido la comisión más baja durante el último proceso de licitación. Estos afiliados deberán permanecer en la AFP por un período mínimo. ${ }^{18}$ De esta manera, la AFP ganadora recibirá un flujo constante de participantes por un lapso de dos años, sin tener que incurrir en gastos de marketing o

\footnotetext{
${ }^{15}$ Para un análisis exhaustivo de estas medidas, véase Reyes y Castro (2008).

16 Para una descripción detallada de los retornos mostrados por las AFP sobre sus activos, véase Valdés y Marinovic (2005).

${ }^{17}$ El proyecto de ley enviado al Congreso incluía una moción para permitir a los bancos locales ingresar a la industria de AFP creando firmas subsidiarias. Esta medida no fue aprobada por los partidos de la oposición en parte como una forma de evitar la creación de una AFP estatal como subsidiaria del Banco Estado.

${ }^{18} \mathrm{El}$ afiliado puede cambiarse a otra administradora si la AFP ganadora no cumple con la reglamentación o presenta un desempeño inferior a otras administradoras que no puede ser compensado por la diferencia en el valor de la comisión.
} 
de personal de ventas. Esta medida crea un atractivo punto de partida para potenciales entrantes, puesto que las firmas incumbentes no pueden cobrar una comisión diferente a distintos grupos de participantes (afiliados actuales o nuevos trabajadores).

\section{- Estructura de comisiones}

Otra explicación de la baja sensibilidad de la demanda, especialmente en relación con las comisiones cobradas, es la complejidad para comparar tarifas entre las distintas administradoras, las que pueden cobrar sus honorarios de diferente manera (una parte proveniente de una tarifa fija y otra originada en un porcentaje fijo del ingreso imponible). En un intento de facilitar la comparación de precios entre las AFP, en la reforma se simplificó la estructura de comisiones al punto de que las AFP solo pueden cobrar una comisión única, expresada como un porcentaje fijo del ingreso imponible.

- Requerimiento o facilidades para la subcontratación de algunas funciones de la AFP

Respecto de la oferta, una serie de medidas tienden a facilitar la tercerización de algunas funciones de las AFP. El rango de servicios que pueden ser subcontratados es ampliado significativamente. Uno de estos servicios, el sis, debe ahora ser contratado por el sistema de AFP en su conjunto, a diferencia de la situación previa en que cada AFP debía tomar su propio seguro y los contratos eran diseñados de manera tal que la mayor parte del riesgo recaía en la propia AFP. ${ }^{19}$ Esto creó un poderoso incentivo para competir en cuanto a la capacidad de atraer selectivamente individuos de bajo riesgo, en detrimento de un buen manejo de las inversiones, de la reducción de costos o de una mejor calidad de servicio. Mediante el requisito de suscribir un contrato sistémico de seguro, el riesgo recae efectivamente en compañías aseguradoras y el incentivo a "descremar" individuos riesgosos es eliminado. El diseño de la licitación del sis estará sujeto a una regulación detallada por parte de las superintendencias de Pensiones y de Valores y Seguros. Por ley, la cobertura del seguro deberá ser licitada separadamente para hombres y mujeres, pudiendo formarse grupos de afiliados elegidos aleatoriamente y asignados a diferentes firmas a fin de evitar una concentración excesiva de los riesgos.

\footnotetext{
${ }^{19}$ Los contratos de seguro incluían ajustes ex post que equivalían a una transferencia del riesgo entre la compañía aseguradora y la AFP, relegando la cobertura provista por el seguro solo para casos extremos.
}

d) Flexibilización del régimen de inversiones de las AFP.

Con el propósito de limitar la exposición absoluta de las carteras de inversión, en la regulación original se incluía un complejo conjunto de límites cuantitativos: por emisor, por emisión, por tipo de activo (incluidos los límites a la renta variable), por origen de los fondos (nacional o extranjero), entre otros. La mayoría de estos límites estaban comprendidos en la propia ley que regulaba el sistema, dando escaso margen para la interpretación o la flexibilidad. Con la reforma, la mayoría de estos límites fueron trasladados a regulaciones secundarias y se creó un consejo especial de inversiones (el Consejo Técnico de Inversiones) cuya función es realizar recomendaciones con respecto a las políticas de inversión y regulación de las AFP. ${ }^{20}$

La mayor flexibilidad será acompañada del incremento en los requisitos de transparencia, en términos de políticas explícitas de inversión y de resolución de conflictos de interés. En la ley reformada se incluye la posibilidad de establecer límites basados en indicadores del riesgo de la cartera de inversiones, en lugar de límites cuantitativos por tipo de activos.

\section{e) Efectos esperados y sostenibilidad fiscal de la reforma}

Dado que los beneficios del Nuevo Pilar Solidario fueron planteados como derechos de la población, la reforma implica un compromiso significativo del Estado respecto de las generaciones futuras de pensionados. Aunque la información detallada sobre la repercusión fiscal de la reforma en el mediano y largo plazo es limitada, los datos disponibles indican que el costo puede ser relevante. El proyecto de ley de reforma fue acompañado de un informe financiero, que incluye estimaciones de costo fiscal entre los años 2008 y 2025. En el cuadro 2 se presentan los efectos esperados de todas las provisiones incorporadas en la ley reformada, incluidas algunas que pueden no ser consideradas como parte de la reforma del sistema previsional en un sentido estricto. Las proyecciones señalan que el costo fiscal de la reforma debiera ser inferior al $0,5 \%$ del PIB durante

\footnotetext{
${ }^{20}$ Solo los principales límites estructurales permanecieron en la ley, sujetos a un límite superior bajo el cual el Banco Central de Chile tiene la autoridad para fijar los límites reales: un límite de renta variable por tipo de fondo; un límite a la inversión extranjera total (que puede alcanzar al 80\% de los fondos) sustituible por límites específicos por tipo de fondo; límites específicos por fondo respecto de la suma de inversiones no cubiertas en moneda extranjera; y finalmente, un límite a las inversiones en instrumentos financieros emitidos por instituciones con menos de tres años en operación.
} 
los primeros años, para alcanzar aproximadamente al $1 \%$ del PIB hacia el año $2025 .^{21}$

\section{- Resultados preliminares del Nuevo Pilar Solidario}

Los beneficios del nuevo Sistema de Pensiones Solidarias comenzaron a pagarse a partir del 1 de julio del año 2008 (Fajnzylber, 2010). En un comienzo, solo se empezaron a pagar las pensiones básicas solidarias (PBS) tanto de vejez como de invalidez, provenientes en su mayoría del sistema de pensiones asistenciales de vejez e invalidez. ${ }^{22}$ A partir de octubre de 2008, se comenzaron a pagar aportes previsionales solidarios (APS), aunque estos representan todavía una pequeña fracción del total de beneficios.

Como se muestra en el cuadro 1, durante septiembre de 2009 se pagaron 623.296 subsidios solidarios, de los cuales el $95 \%$ correspondió a PBS de vejez (62\%) e invalidez (33\%). El 64\% de los beneficios fueron pagados a mujeres.

\footnotetext{
${ }^{21}$ Para mayores detalles, véase Dirección de Presupuestos (2008).

22 Entre enero y junio de 2008 se pagaron en promedio 228.065 pensiones asistenciales de vejez y 212.327 de invalidez (www.suseso. cl). Nótese que algunas de las pensiones de invalidez eran pagadas a personas mayores de 65 años, por lo que las cifras no son directamente comparables con las pensiones básicas solidarias de vejez e invalidez que comenzaron a pagarse a partir de julio de 2008 .
}

En relación con la escasa importancia relativa de los APS con respecto a las PBS, se estima que este es un hecho transitorio originado en los siguientes fenómenos:

i) La mayor parte de los actuales beneficiarios de PBS proviene de beneficiarios de pensiones asistenciales. Estos, a su vez, provienen en cierta medida de afiliados que se pensionaron, agotaron su saldo y se acogieron al beneficio asistencial. Una vez que el sistema se encuentre en régimen, los afiliados de bajo saldo se acogerán a un beneficio de APs desde un principio.

ii) En forma transitoria, los afiliados que al entrar en vigencia la reforma tenían más de 65 años y se encontraban pensionados en retiro programado pueden acogerse al beneficio de APS cuando lo deseen, calculándose el beneficio sobre la base del saldo en el momento de realizar la solicitud. Esto implica que muchos individuos con bajo saldo en sus cuentas pueden preferir mantenerse ajustados a la pensión mínima (actualmente cercana a los $\$ 105.000$ ) hasta agotar su saldo y entonces solicitar el beneficio de APS (que equivaldría a un beneficio de PBS de $\$ 75.000$, pues la persona no tendría saldo). ${ }^{23}$ Este proceso ocurrirá paulatinamente, lo

${ }^{23}$ A modo de ejemplo, una persona cuya pensión según cálculo en retiro programado equivaliera a $\$ 30.000$ podría optar por solicitar el beneficio de APS, en cuyo caso recibiría $\$ 96.000$ por el resto de su

Número de PBS y APS pagadas por mes, entre julio de 2008 y septiembre de 2009

\begin{tabular}{|c|c|c|c|c|c|c|c|c|c|c|c|c|}
\hline & \multicolumn{4}{|c|}{ Hombres } & \multicolumn{4}{|c|}{ Mujeres } & \multicolumn{4}{|c|}{ Total } \\
\hline & $\begin{array}{c}\text { PBS } \\
\text { Vejez }\end{array}$ & $\begin{array}{c}\text { PBS } \\
\text { Invalidez }\end{array}$ & $\begin{array}{c}\text { APS } \\
\text { Vejez }\end{array}$ & $\begin{array}{c}\text { APS } \\
\text { Invalidez }\end{array}$ & $\begin{array}{c}\text { PBS } \\
\text { Vejez }\end{array}$ & $\begin{array}{c}\text { PBS } \\
\text { Invalidez }\end{array}$ & $\begin{array}{c}\text { APS } \\
\text { Vejez }\end{array}$ & $\begin{array}{c}\text { APS } \\
\text { Invalidez }\end{array}$ & $\begin{array}{c}\text { PBS } \\
\text { Vejez }\end{array}$ & $\begin{array}{c}\text { PBS } \\
\text { Invalidez }\end{array}$ & $\begin{array}{c}\text { APS } \\
\text { Vejez }\end{array}$ & $\begin{array}{c}\text { APS } \\
\text { Invalidez }\end{array}$ \\
\hline julio-2008 & 101418 & 84277 & - & - & 189152 & 108433 & - & - & 290570 & 192710 & - & - \\
\hline agosto-2008 & 101669 & 84831 & - & - & 190467 & 109473 & - & - & 292136 & 194304 & - & - \\
\hline septiembre-2008 & 108162 & 84559 & - & - & 221383 & 108959 & - & - & 329545 & 193518 & - & - \\
\hline octubre-2008 & 110075 & 84437 & 955 & 229 & 230808 & 108604 & 2444 & 217 & 340883 & 193041 & 3399 & 446 \\
\hline noviembre-2008 & 111136 & 84286 & 1159 & 294 & 236178 & 108404 & 3067 & 290 & 347314 & 192690 & 4226 & 584 \\
\hline diciembre-2008 & 113630 & 84823 & 1234 & 348 & 244815 & 109628 & 3251 & 352 & 358445 & 194451 & 4485 & 700 \\
\hline enero-2009 & 114748 & 85291 & 1765 & 481 & 249383 & 110901 & 4434 & 468 & 364131 & 196192 & 6199 & 949 \\
\hline febrero-2009 & 115183 & 85481 & 2132 & 584 & 251808 & 111775 & 5048 & 596 & 366991 & 197256 & 7180 & 1180 \\
\hline marzo-2009 & 115759 & 85609 & 2584 & 703 & 254074 & 112459 & 5703 & 787 & 369833 & 198068 & 8287 & 1490 \\
\hline abril-2009 & 116177 & 85787 & 3131 & 922 & 255795 & 113104 & 6499 & 1119 & 371972 & 198891 & 9630 & 2041 \\
\hline mayo-2009 & 116638 & 86085 & 3842 & 1125 & 258094 & 114003 & 7208 & 1372 & 374732 & 200088 & 11050 & 2497 \\
\hline junio-2009 & 116982 & 86441 & 4373 & 1346 & 260086 & 114983 & 7817 & 1674 & 377068 & 201424 & 12190 & 3020 \\
\hline julio-2009 & 117124 & 86710 & 4882 & 1670 & 261334 & 116028 & 8395 & 2118 & 378458 & 202738 & 13277 & 3788 \\
\hline agosto-2009 & 117348 & 87229 & 5603 & 1969 & 261917 & 117514 & 9052 & 2469 & 379265 & 204743 & 14655 & 4438 \\
\hline septiembre-2009 & 117430 & 87678 & 15010 & 2203 & 267244 & 118761 & 12193 & 2777 & 384674 & 206439 & 27203 & 4980 \\
\hline
\end{tabular}

Fuente: elaborado a partir de información disponible en www.spensiones.cl

PBS: Pensión Básica Solidaria.

APS: Aporte Previsional Solidario. 
que debiera aumentar en forma gradual el número de beneficiarios de APS.

Con respecto a los montos de los beneficios, la ley estableció que en un principio los beneficios de PBS corresponderían a $\$ 60.000$ mensuales. Estos fueron aumentados a $\$ 75.000$ mensuales a partir de julio de 2009. En el cuadro 2 se muestran los montos promedio de beneficios pagados en cada mes, por tipo de beneficio y sexo del beneficiario. ${ }^{24}$

Cabe destacar que en principio los beneficios promedio de APS debieran ser siempre inferiores a los de PBS. En el cuadro 2 esto no ocurre debido a la

vida u optar por mantenerse ajustado a la pensión mínima (\$105.000) hasta agotar el saldo (lo que ocurriría en cerca de cuatro años) y recibir la PBS desde ese momento en adelante (\$75.000).

${ }_{24}$ Vale la pena destacar que en los montos promedio se incluyen pagos efectuados en forma retroactiva desde el momento de ingreso de la solicitud. A modo de ejemplo, si una persona solicitó un beneficio de APS el 1 de julio de 2008 y su primer pago se efectuó en octubre de 2008, dicho pago debiera incluir el monto correspondiente a cuatro pagos. Ello explica por qué los promedios son superiores al beneficio máximo ( $\$ 60.000$ antes de julio de 2009 y $\$ 70.000$ a partir de ese momento) y en los primeros meses de pago de APs se registran montos promedio muy superiores al beneficio máximo. inclusión de primeros pagos, que pueden comprender más de un pago mensual y por lo tanto suelen ser superiores. Esta situación debería normalizarse a lo largo del tiempo.

\section{- Desafios pendientes}

El aspecto más importante de la reforma descrita consiste en que en lugar de reemplazar al sistema de AFP creado en 1980, lo mejora integrando un pilar de prevención de la pobreza financiado por el Estado, extendiendo el pilar voluntario a los trabajadores de ingreso medio e introduciendo una serie de medidas para mejorar la cobertura y la competencia en el mercado de las AFP. Esto es resultado de un largo proceso participativo de dos años, precedido de una investigación exhaustiva y de diversos esfuerzos de evaluación.

Aún persiste una serie de desafíos que deberán ser abordados en los próximos años, relacionados tanto con la implementación de la reforma como con aspectos de más largo plazo. En la primera categoría, la aplicación progresiva del Nuevo Pilar Solidario probablemente enfrentará riesgos. Por una parte, no se conoce claramente el número real de potenciales beneficiarios, ya que ello depende de la evolución de los salarios, el cumplimiento de la normativa y el retorno

Monto promedio de PBS y APS por mes

(Equivalente en dólares ${ }^{\mathrm{a}}$ )

\begin{tabular}{|c|c|c|c|c|c|c|c|c|c|c|c|c|}
\hline & \multicolumn{4}{|c|}{ Hombres } & \multicolumn{4}{|c|}{ Mujeres } & \multicolumn{4}{|c|}{ Total } \\
\hline & $\begin{array}{c}\text { PBS } \\
\text { Vejez }\end{array}$ & $\begin{array}{c}\text { PBS } \\
\text { Invalidez }\end{array}$ & $\begin{array}{c}\text { APS } \\
\text { Vejez }\end{array}$ & $\begin{array}{c}\text { APS } \\
\text { Invalidez }\end{array}$ & $\begin{array}{c}\text { PBS } \\
\text { Vejez }\end{array}$ & $\begin{array}{c}\text { PBS } \\
\text { Invalidez }\end{array}$ & $\begin{array}{c}\text { APS } \\
\text { Vejez }\end{array}$ & $\begin{array}{c}\text { APS } \\
\text { Invalidez }\end{array}$ & $\begin{array}{c}\text { PBS } \\
\text { Vejez }\end{array}$ & $\begin{array}{c}\text { PBS } \\
\text { Invalidez }\end{array}$ & $\begin{array}{c}\text { APS } \\
\text { Vejez }\end{array}$ & $\begin{array}{c}\text { APS } \\
\text { Invalidez }\end{array}$ \\
\hline julio-2008 & 120 & 120 & & & 120 & 120 & & & 120 & 120 & & \\
\hline agosto-2008 & 120 & 120 & & & 120 & 120 & & & 120 & 120 & & \\
\hline septiembre-2008 & 120 & 120 & & & 120 & 120 & & & 120 & 120 & & \\
\hline octubre-2008 & 126 & 121 & 401 & 314 & 132 & 120 & 316 & 326 & 130 & 121 & 340 & 320 \\
\hline noviembre-2008 & 124 & 121 & 148 & 134 & 128 & 121 & 126 & 142 & 127 & 121 & 132 & 138 \\
\hline diciembre-2008 & 129 & 125 & 175 & 116 & 132 & 129 & 122 & 120 & 131 & 127 & 137 & 118 \\
\hline enero-2009 & 124 & 125 & 221 & 182 & 125 & 130 & 174 & 163 & 125 & 128 & 188 & 172 \\
\hline febrero-2009 & 123 & 124 & 147 & 150 & 124 & 129 & 118 & 158 & 124 & 127 & 126 & 154 \\
\hline marzo-2009 & 123 & 124 & 156 & 135 & 123 & 129 & 116 & 157 & 123 & 127 & 129 & 147 \\
\hline abril-2009 & 122 & 125 & 180 & 173 & 122 & 129 & 146 & 195 & 122 & 127 & 157 & 185 \\
\hline mayo-2009 & 122 & 126 & 172 & 148 & 123 & 131 & 121 & 151 & 122 & 129 & 139 & 150 \\
\hline junio-2009 & 122 & 127 & 140 & 140 & 123 & 133 & 113 & 147 & 123 & 130 & 122 & 144 \\
\hline julio-2009 & 152 & 157 & 174 & 207 & 152 & 164 & 151 & 210 & 152 & 161 & 160 & 209 \\
\hline agosto-2009 & 154 & 159 & 207 & 183 & 152 & 168 & 163 & 187 & 153 & 164 & 180 & 185 \\
\hline septiembre-2009 & 152 & 159 & 132 & 169 & 158 & 166 & 157 & 173 & 156 & 163 & 143 & 172 \\
\hline
\end{tabular}

Fuente: elaborado a partir de información disponible en www.spensiones.cl

PBS: Pensión Básica Solidaria.

APs: Aporte Previsional Solidario.

a En el cálculo de la equivalencia se utilizó un tipo de cambio de \$ 500 por dólar. 
de los fondos de pensiones. Asimismo, organizar las nuevas instituciones, crear las condiciones para la integración completa de los trabajadores independientes al sistema, e implementar los sistemas para identificar a los beneficiarios de las nuevas prestaciones solidarias y realizar los pagos, demandará un compromiso serio por parte del gobierno.

Respecto de los desafíos de más largo plazo, no necesariamente abordados por las reformas recientes, el más importante es compartido por la mayoría de los países de medianos y altos ingresos: el aumento de la esperanza de vida y los costos médicos de los adultos mayores. El progreso tecnológico de las últimas décadas ha implicado un incremento en la esperanza de vida, basado en tratamientos y equipamiento de creciente sofisticación. Si bien existe una idea razonable acerca de cuánto van a vivir los pensionados actuales, poco se sabe sobre la esperanza de vida de los individuos que están ingresando al mercado de trabajo. Es probable que la actual tasa de contribución de $10 \%$ del ingreso imponible sea insuficiente para financiar beneficios en el futuro y no es claro que las diferencias generacionales permitan a los trabajadores permanecer en el mercado de trabajo el tiempo suficiente para compensar este aumento. La mayor parte del peso recaerá en la capacidad de los individuos para prever estos déficits y acrecentar sus ahorros voluntarios. Dado que los sistemas de pensiones fueron creados para morigerar las tensiones de corto plazo, no resulta obvio que esta reacción voluntaria pueda tener el timing y la fuerza adecuados. Mayor esfuerzo deberá ponerse en mejorar el conocimiento sobre este incierto futuro y, en consecuencia, adoptar las —usualmente impopulares - medidas de incremento de las tasas de cotización o de la edad mínima de retiro.

\section{IV}

\section{Algunas notas sobre los procesos políticos detrás de las reformas}

Hasta aquí, en este trabajo se han analizado las reformas recientes afrontadas por los sistemas de pensiones de la Argentina y Chile. Alcanzado este punto, se pretende aportar una breve nota sobre la relevancia de los procesos políticos subyacentes al trazado de la política jubilatoria discutida previamente. El interés de los autores en este aspecto se apoya en el hecho de que algunos elementos sustantivos, que definen el funcionamiento de los sistemas previsionales argentino y chileno, parecen originarse en las características de las instituciones políticas que los diseñan y gestionan, y no tanto en problemas "técnicos" inherentes a ellos.

Indudablemente, las consideraciones sobre la relevancia y las consecuencias de las instituciones políticas y los procesos de elaboración de políticas públicas (policymaking) trascienden a la política previsional. En una parte significativa de la actual literatura sobre políticas públicas se aborda la idea de que estas requieren cierta capacidad de policymaking para ser efectivas. Específicamente, se entiende que diversos aspectos significativos de las políticas públicas dependen de la habilidad para consolidar acuerdos políticos intertemporales (Spiller, Stein y Tommasi, 2003). En el caso de la política previsional, lo anterior se ve agudizado por una serie de factores que la distinguen. Se trata de una política explícitamente distributiva, con objetivos múltiples que entrañan costos de oportunidad entre ellos, y que se desenvuelve en una secuencia de tiempo que la torna única. Si se tiene en cuenta que las transferencias involucradas son cuantiosas, que todo sistema jubilatorio - al organizar derechos sobre el producto social futurose basa ineludiblemente en algún tipo de promesas (Barr, 2002) y que la duración integral del ciclo de la política previsional es absolutamente excepcional, podrá deducirse la importancia determinante de las dinámicas políticas que se articulan a su alrededor.

Puede señalarse —en una muy apretada síntesisque la configuración institucional de la Argentina ha sido frecuentemente juzgada como desfavorable y adversa para la consecución y el sustento de comportamientos políticos cooperativos, habiéndose caracterizado, en cambio, por actores políticos que han enfrentado cortos horizontes temporales o incentivos inadecuados o bien ambos factores a la vez (Spiller, Stein y Tommasi, 2003). Por otra parte, desde el retorno a la democracia, Chile parece haber mostrado mecanismos más sólidos en sus procesos de policymaking. Los cambios en las políticas han sido incrementales y, por lo general, han surgido como resultado de un proceso relativamente intenso e institucionalizado. En suma, en Chile parecen haberse 
encontrado dinámicas de policymaking que tienden a facilitar las respuestas cooperativas en el juego de transacciones políticas.

Si volvemos nuestra atención hacia la política de pensiones, es posible señalar que el recorrido de las recientes reformas en la Argentina y Chile se presenta consistente con este diagnóstico dispar. En efecto, las últimas reformas ofrecen un abanico de circunstancias donde la capacidad de articulación de intereses y el compromiso político intertemporal parecen haberse manifestado en forma desigual. Remontémonos a las reformas estructurales efectuadas en Chile (1980) y en la Argentina (1993) como un punto de partida semejante para analizar los procesos más recientes descritos con anterioridad. Ambas reformas fueron presentadas, en su tiempo, como "íconos" de procesos de reforma más amplios. En Chile, la reforma jubilatoria fue probablemente la más famosa de las autodenominadas "modernizaciones" de la dictadura militar, mientras que en la Argentina constituyó una pieza muy significativa del giro ortodoxo protagonizado por el primer gobierno de Menem. Así, en tanto "íconos", las reformas estuvieron rodeadas de una notable batalla comunicacional entre promotores y oponentes de lo que terminaría denominándose la "privatización" de las jubilaciones.

Pero en la Argentina la reforma de 1993 no eliminó por completo el viejo sistema; la ley aprobada por el Congreso de la Nación, a diferencia del sistema chileno y de la propuesta original del Ejecutivo argentino, no clausuró definitivamente el esquema de reparto, sino que fundó un modelo multipilar. Este hecho no parece haber sido trivial: desde el momento mismo de la introducción de la reforma y hasta el presente, bajo el reciente espíritu "revisionista" que ha estado rondando, el debate general sobre el sistema de pensiones ha quedado (casi exclusivamente) atrapado bajo la dicotomía simplista de "lo privado" versus "lo público". En el momento de introducir aquella reforma, las autoridades políticas promovían explícitamente al nuevo régimen de cuentas individuales como "lo nuevo" en materia jubilatoria y alentaban a la gente a unirse a él, mientras que la inexistencia de una estrategia informativa seria y objetiva dirigida a los trabajadores en situaciones dispares resultaba flagrante (Isuani y San Martino, 1995). Desde hace unos años, en cambio, la tendencia discursiva en boga giró drásticamente hacia la segunda de estas posiciones; de hecho, el principal mensaje transmitido por las autoridades en relación con las últimas reformas consistió en que los trabajadores reconquistarían la posibilidad de trasladarse desde el sistema "privado" al "público" y, finalmente, que la reunificación bajo un esquema público de reparto generaría mejores beneficios a los jubilados. Notoriamente, ni entonces ni ahora parecieron existir canales políticos adecuados que - trascendiendo el debate superficial y mecanicista sobre aquella falsa dicotomía- le den forma a un diálogo social más profundo y sistemático que procure adentrarse en las (muy complejas) dinámicas de negociación y cooperación intertemporal, requeridas por todo sistema jubilatorio que se pretenda eficiente, inclusivo y sostenible.

Por su parte, en Chile, una vez en el poder y tal como sucediera en otras áreas de política pública, los gobiernos de la Concertación optaron por la continuidad frente al cambio radical y decidieron así apuntalar el sistema de pensiones reformado. Desde 1990 las reformas al sistema habían sido menores, principalmente dirigidas a modificar las regulaciones de inversión. En años recientes, la cuestión de la cobertura emergió como elemento crítico y se transformó en el centro de los debates de política. En ese sentido, en marzo de 2006 se creó desde el Ejecutivo el mencionado Consejo Asesor para la Reforma Previsional, destinado a analizar la evolución del sistema, estudiar sus déficits y desarrollar un proceso de audiencias públicas que duraría 90 días. El Consejo, conformado por prestigiosos especialistas en el tema, proporcionó un diagnóstico profundo y propuso una serie de reformas sustantivas. A continuación, el gobierno estableció un Comité de Ministros para evaluar las recomendaciones del Consejo, de lo cual resultó finalmente un proyecto de reforma enviado al Congreso y aprobado en enero de 2008.

En contraposición, las reformas más recientes del sistema argentino, analizadas en detalle en la sección II, surgieron de un proceso desarrollado a puertas cerradas, donde unas pocas figuras políticas definieron el camino a recorrer a través de una serie de pasos sucesivos (y no siempre coordinados) en que prácticamente se excluyó a los diversos referentes principales del tema previsional. Como se vio anteriormente, la moratoria para autónomos, que finalmente se tornó de una masividad sin precedentes, constituyó una de las reformas más significativas de los últimos años en el terreno previsional argentino. Esta fue establecida a partir de una serie combinada de leyes y decretos que condujeron a un incremento masivo en el número de beneficiarios. El proceso institucional subyacente a una reforma de tal importancia fue sumamente particular: por ejemplo, el proyecto de ley que derivó en la última modificación que disparó la masividad de la moratoria, se originó en el Congreso de la Nación y fue aprobado sin discusión, esto es, sin que mediara 
debate alguno entre los legisladores, quienes votaron el dictamen tal como surgió de las comisiones. De esta forma, la nueva ley no captó la atención de la prensa ni de las autoridades políticas y, notablemente, no existió tampoco un anuncio o acto formal de lanzamiento de la iniciativa. Un año más tarde, a partir de un decreto dictado en noviembre de 2008 que regulaba el proceso, el programa se tornó operativo. A su vez, la posterior eliminación del componente de capitalización individual con gestión privada y su reemplazo por un sistema público de reparto reunificado fueron anunciados en forma sorpresiva a finales de octubre de 2008 y transformados en ley en diciembre de ese año. La ley eliminó el esquema de capitalización y dispuso que todos los contribuyentes, beneficiarios y activos afiliados a él fueran transferidos al sistema público de reparto. Una vez más, el debate del proyecto en el Congreso fue sumamente breve, debido a que este recibió un rápido apoyo desde diversos sectores políticos.

En definitiva, es posible identificar un patrón más o menos claro en el trazado de la política jubilatoria reciente en ambos países. De acuerdo con las

\section{$\mathrm{V}$}

\section{Conclusiones}

Dentro de la región, la Argentina y Chile han sido tradicionalmente dos países pioneros en el avance de las políticas sociales. Ambos están dentro del pequeño grupo de países que introdujeron sistemas jubilatorios a principios del siglo XX y, a partir de entonces, avanzaron ofreciendo progresivamente cobertura a un mayor número de trabajadores. En 1980, se tomaron nuevamente en Chile medidas precursoras al introducir una reforma estructural que, entre otros cambios importantes, instauraba una gestión privada de los fondos de pensión. En la Argentina, con ciertas diferencias, se siguió el modelo chileno una década después cuando el tradicional esquema de reparto fue transformado en un esquema multipilar.

El adelantamiento relativo en la adopción de reformas continuó en los años recientes, en tanto ambos países introdujeron, una vez más, importantes cambios en sus sistemas. Estos comparten claramente algunos objetivos comunes, tales como la expansión de la cobertura en la vejez y la redefinición del papel del Estado en facilitar el acceso a los beneficios. Sin embargo, existieron significativas diferencias en otros aspectos, incluida la organización institucional de los características de los sistemas jubilatorios mencionados anteriormente, las reformas previsionales han sido identificadas en su necesidad de requerir no solo un minucioso análisis técnico, sino también de contar con un proceso de recolección y difusión de información precisa para obtener apoyo y consenso (BID, 2007). En las reformas previsionales recientes, Chile parece haber logrado acercarse más a esos estándares que la Argentina, donde los cambios introducidos se sucedieron de una manera desordenada, mostrando un escaso nivel de coherencia entre sí. Muchos de los anuncios más importantes fueron completamente sorpresivos y tuvieron un muy rápido trámite en el Congreso. A diferencia de lo apuntado para el caso chileno, en las reformas argentinas no se observaron grandes esfuerzos por plantear un debate amplio y un examen cuidadoso que incluyera a los diferentes actores involucrados (directa o indirectamente) en el campo previsional. Tampoco se desarrollaron una consideración y discusión detalladas con respecto a las implicancias fiscales presentes y futuras de las distintas medidas que se estaban llevando a cabo.

sistemas, en parte debido a ciertos diferentes enfoques de criterio político sobre la operatoria de las estructuras previas y la implementación de procesos dispares en el policymaking de ambos países.

Las reformas en la Argentina se tradujeron en un incremento inmediato de la cobertura, estableciéndose un brusco quiebre respecto de la tendencia previa. El número total de jubilados se elevó en más del $70 \%$ en dos años, debido a la introducción de una moratoria que devino en un programa de inclusión masiva. La moratoria en cuestión permitía que cualquier individuo que estuviera por sobre la edad establecida de retiro pudiera aplicar para obtener un beneficio jubilatorio, con independencia de su registro de contribuciones pasadas e, incluso, de su potencial carácter de beneficiario efectivo de algún otro tipo de pensión ya vigente. ${ }^{25}$ Las reformas también implicaron la anulación de una parte

\footnotetext{
${ }^{25}$ A medida que el programa avanzaba, se introdujo una restricción para evitar la duplicación de beneficios, aunque esta no incluía límites respecto de aquellos que recibían una pensión por supervivencia.
} 
central de los cambios introducidos en 1993, en tanto se eliminaron las cuentas de capitalización individual y se retornó a una operatoria unificada bajo la gestión de una agencia pública montada según un sistema que vuelve a operar sobre una base de beneficios definidos. Se terminó así con la existencia de las administradoras privadas de fondos de pensiones. Sin embargo, resulta importante señalar que los cambios no representaron un retorno completo a la situación previa a 1993, dado que la mayoría de los parámetros del sistema (incluidas las tasas de contribución vigentes, las edades de retiro y las tasas de reemplazo) no fueron retrotraídos a los antiguos niveles. Asimismo, la agencia pública encargada de gestionar el nuevo sistema reunificado continuará recibiendo una porción de la recaudación de impuestos generales (originalmente asignada para financiar los costos de transición). Por consiguiente, resulta esperable que esta agencia maneje fondos crecientes: en 2008 estos llegaron a representar más del 10\% del PIB argentino. Finalmente, y hasta hoy, no ha existido estimación oficial alguna con respecto al impacto fiscal que representan las reformas, ya sea en el corto o en el mediano plazo.

En Chile, a su vez, la mayoría de las reformas mostrarán sus efectos de manera gradual en el tiempo. El número de beneficiarios del Nuevo Pilar Solidario será limitado en un principio, pero aumentará gradualmente a medida que avance la implementación del sistema. Sin duda, esta es la novedad principal de las reformas recientemente efectuadas en tanto estas deberían conducir a un escenario de cobertura universal en un futuro cercano. Otro conjunto de reformas afectan a aspectos operativos del sistema existente y a la estructura institucional de las agencias gubernamentales de supervisión y control. Asimismo, una serie de cambios apuntaron a eliminar ciertas inequidades del sistema, sobre todo las atinentes a las diferencias de género.
El diseño y la aprobación de estas reformas siguieron senderos sumamente diferentes en ambos países, lo que fue un reflejo de los dispares contextos institucionales vigentes. La reforma en Chile se inició cuando la Presidenta Bachelet anunció su intención de hacerlo y designó a un Consejo de expertos. Dos años después, tras diversos debates, publicaciones y análisis, la reforma fue aprobada. En la Argentina, la mayoría de las decisiones se hicieron efectivas de manera muy rápida al mayor nivel de decisión del Ejecutivo, y el proceso de consulta y discusión fue muy breve y limitado. Estas divergencias pueden contribuir a explicar diferencias en los resultados y, por tanto, son merecedoras de un estudio y análisis más detallado en el futuro.

Es probable que el proceso más pausado y cuidadoso adoptado por las autoridades chilenas para realizar los cambios previsionales contribuirá a alcanzar resultados más sostenibles en el tiempo. Sin embargo, la ejecución de una reforma más acelerada en la Argentina alcanzó a dar una respuesta inmediata al gran problema que representaba la disminución masiva de la cobertura. La mayoría de los adultos mayores excluidos del sistema recibieron un beneficio jubilatorio en menos de un año, alcanzando una mejora inmediata en relación con su situación previa; en Chile, en cambio, el proceso que implica alcanzar a dar cobertura a todos los beneficiarios considerados será más lento y gradual.

Es claro que ninguno de los dos sistemas alcanzó una posición o un diseño que pueda pretenderse "definitivo", en tanto que aún existen desafíos pendientes de política que las autoridades deberán enfrentar en un futuro próximo. Al mismo tiempo, surgirán inevitablemente nuevos problemas y dificultades. La habilidad de los gobiernos futuros para responder adecuadamente a estos dilemas resultará decisiva a la hora de definir el bienestar de las nuevas generaciones de chilenos y argentinos.
ANSES (Administración Nacional de la Seguridad Social) (2009), Informe de la seguridad social. Cuarto trimestre 2009, Buenos Aires.

Arenas de Mesa, Alberto (2000), "Cobertura previsional en Chile: lecciones y desafíos del sistema de pensiones administrado por el sector privado", serie Financiamiento del desarrollo, $\mathrm{N}^{\circ} 105$ (LC/L.1457-P), Santiago de Chile, Comisión Económica para América Latina y el Caribe (CEPAL). Publicación de las Naciones Unidas, $\mathrm{N}^{\mathbf{o}}$ de venta: S.00.II.G.137.

Arenas de Mesa, Alberto y Fabio Bertranou (1997), "Learning from social security reforms: two different cases, Chile and Argentina", World Development, vol. 25, № 3, Amsterdam, Elsevier.

Arenas de Mesa, Alberto y otros (2006), "The Chilean pension reform turns 25: lessons from the social protection survey",
NBER Working Paper, $\mathrm{N}^{\circ} \mathrm{W} 12401$, Cambridge, Massachusetts, National Bureau of Economic Research.

Banco Mundial (1994), Averting the Old Age Crisis: Policies to Protect the Old and Promote Growth, Nueva York, Banco Mundial.

Barr, Nicholas (2002), "The pension puzzle: prerequisites and policy choices in pension design", Economic Issues, $\mathrm{N}^{\circ} 29$, Washington, D.C., Fondo Monetario Internacional.

Berstein, Solange (2007), El sistema chileno de pensiones, Santiago de Chile, Superintendencia de Administradoras de Fondos de Pensiones.

Berstein, Solange, Guillermo Larraín y Ariel Pino (2006), "Chilean pension reform: coverage facts and policy alternatives", Economía, vol. $6, \mathrm{~N}^{\circ}$ 2, Baltimore, Brookings Institution Press. 
BID (Banco Interamericano de Desarrollo) (2007), "Chile: Strengthening of Pension System Management and Information. Report of the Institutional Capacity Assessment System (ICAS)", Washington, D.C.

CEPAL (Comisión Económica para América Latina y el Caribe) (2006), La protección social de cara al futuro: acceso, financiamiento y solidaridad (LC/G.2294(SES.31/3)), Santiago de Chile, febrero.

Consejo Asesor Presidencial para la Reforma Previsional (2006), El derecho a una vida digna en la vejez, Santiago de Chile.

Corbo, Vittorio y Klaus Schmidt-Hebbel (2003), Efectos macroeconómicos de la reforma de pensiones en Chile. Resultados y desafios de las reformas a las pensiones, Santiago de Chile, Federación Internacional de Administradoras de Fondos de Pensiones (FIAP).

Dirección de Presupuestos (2008), "Informe financiero sustitutivo", Informe financiero, $\mathrm{N}^{\circ}$ 01/2008, Santiago de Chile, Ministerio de Hacienda.

Fajnzylber, Eduardo (2010), "Marco metodológico para el análisis del sistema de pensiones solidarias", Informe elaborado para el Banco Interamericano de Desarrollo, enero.

(2006), "Pensiones para todos: análisis de alternativas para extender la cobertura del sistema previsional chileno", En foco, $\mathrm{N}^{\mathrm{o}} 65$ [en línea] http://www.expansivaudp.cl/media/en_foco/ documentos/07032006105928.pdf

Farall, Andrés y otros (2003), "Estudio sobre la frecuencia de los aportes en el SIJP: una primera aproximación hacia las historias laborales", Historias laborales en la seguridad social, Buenos Aires, Ministerio de Trabajo, Empleo y Seguridad Social.

Holzmann, Robert y Richard Hinz (2005), Old-age Income Support in the 21st Century, Washington, D.C., Banco Mundial.

INDEC (Instituto Nacional de Estadística y Censos) (2009), Encuesta Permanente de Hogares Continua. Primer Semestre 2009, Buenos Aires.
Isuani, Aldo y Jorge San Martino (1995), "El nuevo sistema previsional argentino. ¿Punto final a una larga crisis?”, Boletín informativo Techint, $\mathrm{N}^{\mathrm{o}}$ 281-282, Buenos Aires, Techint.

MECON (Ministerio de Economía y Finanzas Públicas) (2009), "Series de gasto público consolidado", Buenos Aires.

Moreno, Juan Martín (2007), “¿Se acuerda, abuelo, cuando las jubilaciones en la Argentina eran Bismarckianas?", documento presentado en la IX Jornada Argentina de Estudios de Población.

Reyes, Gonzalo y Rubén Castro (2008), "Medidas pro-competencia de la reforma previsional", Documento de trabajo, $\mathrm{N}^{\circ} 29$, Santiago de Chile, Superintendencia de AFP.

Rofman, Rafael (2003), "The Pension System and the Crisis in Argentina: Learning the Lessons", Washington, D.C., Banco Mundial.

Rofman, Rafael, Leonardo Lucchetti y Guzmán Ourens (2009), "Pension systems in Latin America: concepts and measurements of coverage", SP Discussion Paper, $\mathrm{N}^{\circ}$ 0616, Washington, D.C., Banco Mundial.

SAFJP (Superintendencia de Administradoras de Fondos de Jubilaciones y Pensiones) (2007), El régimen de capitalización y el sistema previsional, 1994-2007, Buenos Aires.

Santiso, Carlos (2006), "El día que me quieras: parlamentos y presupuestos en América Latina", Cada cual ¿atiende su juego? El rol del Congreso en el presupuesto nacional de Argentina, Buenos Aires, Centro de Implementación de Políticas Públicas para la Equidad y el Crecimiento (CIPPEC).

Spiller, Pablo, Ernesto Stein y Mariano Tommasi (2003), Political Institutions, Policymaking Processes, and Policy Outcomes. An Intertemporal Transactions Framework, Washington, D.C., Banco Interamericano de Desarrollo.

Valdés, Salvador e Iván Marinovic (2005), "Contabilidad regulatoria: las AFP chilenas, 1993-2003”, Documento de trabajo, N² 279 Santiago de Chile, Instituto de Economía, Pontificia Universidad Católica de Chile. 